MATHEMATICS OF COMPUTATION

Volume 68, Number 227, Pages 1025-1055

S 0025-5718(99)01062-5

Article electronically published on February 13, 1999

\title{
GENERALIZED MONOTONE SCHEMES, DISCRETE PATHS OF EXTREMA, AND DISCRETE ENTROPY CONDITIONS
}

\author{
PHILIPPE G. LEFLOCH AND JIAN-GUO LIU
}

\begin{abstract}
Solutions of conservation laws satisfy the monotonicity property: the number of local extrema is a non-increasing function of time, and local maximum/minimum values decrease/increase monotonically in time. This paper investigates this property from a numerical standpoint. We introduce a class of fully discrete in space and time, high order accurate, difference schemes, called generalized monotone schemes. Convergence toward the entropy solution is proven via a new technique of proof, assuming that the initial data has a finite number of extremum values only, and the flux-function is strictly convex. We define discrete paths of extrema by tracking local extremum values in the approximate solution. In the course of the analysis we establish the pointwise convergence of the trace of the solution along a path of extremum. As a corollary, we obtain a proof of convergence for a MUSCL-type scheme that is second order accurate away from sonic points and extrema.
\end{abstract}

\section{INTRODUCTION}

This paper deals with entropy solutions of the Cauchy problem for a onedimensional scalar conservation law:

$$
\begin{gathered}
\partial_{t} u+\partial_{x} f(u)=0, \quad u(x, t) \in \mathbb{R}, t>0, x \in \mathbb{R}, \\
u(x, 0)=u_{0}(x), \quad x \in \mathbb{R},
\end{gathered}
$$

where the flux $f: \mathbb{R} \rightarrow \mathbb{R}$ is a given function of class $C^{2}$ and the initial data $u_{0}$ belongs to the space $B V(\mathbb{R})$ of all integrable functions of bounded total variation. For the main result of this paper, we assume that

$f$ is a strictly convex function

and

$u_{0}$ has a locally finite number of extrema.

Received by the editor May 5, 1997 and, in revised form, November 10, 1997.

1991 Mathematics Subject Classification. Primary 35L65, 65M12.

Key words and phrases. Conservation law, entropy solution, extremum path, monotone scheme, high order accuracy, MUSCL scheme.

The first author was supported in parts by the Centre National de la Recherche Scientifique, and by the National Science Foundation under grants DMS-88-06731, DMS 94-01003 and DMS 95-02766, and a Faculty Early Career Development award (CAREER) from NSF. The second author was partially supported by DOE grant DE-FG02 88ER-25053.

(C)1999 American Mathematical Society 
Solutions to conservation laws are generally discontinuous, and an entropy criterion is necessary to single out a unique solution. We refer the reader to Lax [20], [21] for background on nonlinear hyperbolic equations and the entropy criterion.

As is well-known [19], [37], problem (1.1)-(1.2) admits a unique entropy solution $u$ in $L^{\infty}\left(\mathbb{R}_{+}, B V(\mathbb{R})\right)$ and $\operatorname{Lip}\left(\mathbb{R}_{+} ; L^{1}(\mathbb{R})\right)$. (This result holds without the restriction (1.3)-(1.4) and for multidimensional equations as well.) To select the solution one can use the distributional entropy inequality

$$
\partial_{t} U(u)+\partial_{x} F(u) \leq 0
$$

where the (Lipschitz continuous) function $(U, F): \mathbb{R} \rightarrow \mathbb{R}^{2}$ is a convex entropyentropy flux pair, i.e. $U$ is a convex function and $F^{\prime}=U^{\prime} f^{\prime}$. One can also use the Lax shock admissibility inequality

$$
u(x-, t) \geq u(x+, t)
$$

for all $x$ and $t$. Since $f$ is convex and $u$ has bounded variation, a single entropy $U$ in (1.5) is sufficient to ensure uniqueness, and (1.6) is equivalent to (1.5). At the discrete level, however, conditions (1.5) and (1.6) leads two drastically different notions of consistency with the entropy criterion for a difference scheme. In the present paper we will be using both conditions. Indeed in some regions of the $(x, t)$ plane it is easier to use a discrete version of (1.5), while in other regions (1.6) is more adapted.

We are interested in conservative discretizations of problem (1.1)-(1.2) in the sense of Lax and Wendroff [22]. The monotone schemes and, more generally, the E-schemes are large classes of schemes (including the Godunov and Lax-Friedrichs schemes) for which a convergence analysis is available. The main point is that monotone schemes satisfy a discrete version of the entropy inequality (1.5) (see (2.9) below). However they turn out to be first order accurate only, and so have the disadvantage of introducing a large amount of numerical viscosity that spreads the discontinuities over a large number of computational cells.

The proof of convergence of the monotone schemes and E-schemes is based on Helly's and Lax-Wendroff's theorems. See [3], [6], [8], [11], [16], [26], [31], [35], [23] and the references therein. The result holds even for multidimensional equations and/or when irregular (non-Cartesian) meshes are used.

To get high-order accurate approximations, it is natural to proceed from analytical properties satisfied by the entropy solutions to (1.1), formulate them at the discrete level, and so design large classes of high-order difference schemes. One central contribution in this direction is due to Harten [13], [14], who introduced the concept of TVD schemes, for "Total Variation Diminishing". Harten shows that conservative, consistent, TVD schemes necessarily converge to a weak solution to (1.1). Moreover such schemes possess sharp numerical shock profiles with no spurious oscillations. However, Harten's notion of TVD scheme is weaker than the notion of monotone scheme, and a TVD need not converge to the entropy solution. The aim of this paper is precisely to single out a subclass of TVD schemes, refining Harten's notion, that are both high-order order accurate and entropy satisfying, cf. Definition 2.2 below.

A very large literature is available on the actual design of second-order shockcapturing schemes. One approach to upgrading a first order scheme was proposed by van Leer [23], [24]: the MUSCL scheme extends the Godunov scheme by replacing the piecewise constant approximation in the latter with a piecewise affine 
approximation. The heart of the matter is to avoid the formation of spurious oscillations near discontinuities. This is achieved by van Leer via the so-called min-mod limitor.

Other classes of high order schemes have been built from the maximum principle and a monotony condition: see the classes of ENO and UNO schemes proposed by Harten, Engquist, Osher, and Chakravarthy [15]. In this paper we concentrate attention on the MUSCL scheme, but our main convergence theorem, Theorem 2.3 below, should also be applicable to other schemes.

After Osher's pioneering result [32] and the systematic study of high-order schemes by Osher and Tadmor [33] (which however required a technical condition on the slopes of the affine reconstructions), Lions and Souganidis [27], [28] and, independently, Yang [38] established the convergence of the MUSCL scheme. Both proofs apply to an arbitrary BV initial data (so (1.4) is not assumed); the flux-function is assumed to be convex in [38] and strictly convex in [28]. In both papers significantly new techniques of proof are introduced by the authors. In [38], Yang develops a method for tracking local extremum values. In [28], Lions and Sougadinis elegantly re-formulate the MUSCL scheme at the level of the HamiltonJacobi equation associated with (1.1) and rely on Crandall-Lions' theory of viscosity solutions for such equations. A large class of difference approximations is treated in [28]. Techniques in both papers are restricted in an essential way to semi-discrete schemes, in which the time variable is kept continuous. In [25] the present authors announced a proof of the convergence of a class of fully discrete schemes that include the MUSCL scheme. Independently, Yang [39] also extended his approach to a large class of fully discrete methods.

Several other works deal with the convergence of van Leer's scheme or variants of it. A discrete version of inequality (1.6) was established by Brenier and Osher [2] and Goodman and LeVeque [12], the latter dealing with both first and second order methods. Nessyahu, Tadmor, and Tamir [30] establish both the convergence and error estimates for a variety of Godunov-type schemes. Various approaches to upgrading Lax-Friedrichs scheme are actively developed by Tadmor and coauthors; see, for instance, Nessyahu and Tadmor [29]. An extensive discussion of the discretization of the entropy inequality (1.5) is found in Osher and Tadmor [33] and the many references cited theirein. See also Bouchut, Bourdarias and Perthame [1], and Coquel and LeFloch [4].

The objective of the present paper is to provide a framework to prove the convergence of high order accurate and fully discrete difference schemes. We will strongly rely on a property shared by all entropy solutions to (1.1): the monotonicity property. Given an arbitrary entropy solution $u$, the number of extrema in $u(t)$ is a non-increasing function of $t$, and local maximum/minimum values decrease/increase monotonically in time. (See the Appendix for rigorous statements.) This property was studied first by Harten [13] from the numerical standpoint, in order to arrive at his notion of TVD scheme. It was also essential in [28], [38] and [36].

Observe that monotone or TVD schemes do not necessarily satisfy the monotonicity property. For instance the Lax-Friedrichs scheme may increase the number of extremum values! This motivates the introduction of a subclass of TVD schemes, guaranteeing this property together with the high order of accuracy. The scheme then closely mimics an important property of the solutions to the continuous equation. A further requirement is necessary to ensure that the scheme is entropy-satisfying. 
Based on the monotonicity property, we thus introduce the notion of generalized monotone scheme (Definition 2.2) characterized by three conditions: maximum principle, entropy consistency, and local behavior at extrema. The (first order) Godunov method and the (second order) MUSCL method are prototype examples.

Our first requirement is a (strong) version of the local maximum principle, which will allow us to show a discrete analogue of the monotonicity property. In particular our condition prevents the formation of spurious numerical oscillations, and the scheme is TVD in the sense of Harten.

The second requirement is motivated by the following observation made by Osher for semi-discrete schemes [31]: in the non-decreasing parts of the approximate solutions, it is possible to simultaneously achieve second order accuracy and the existence of one cell entropy inequality -i.e. a discrete analogue of (1.5). Our second condition therefore requires a cell entropy inequality in the non-decreasing regions.

Finally, in order to prevent cusp-like behavior near extrema, which may lead to entropy violating discontinuities, the scheme should be well-behaved near local extrema. To this end, we introduce a condition referred to as the quadratic decay property at local extrema. It reflects the nonlinear behavior of the numerical fluxfunction near extrema, and assumption (1.3) again is essential. For simplicity, it is also assumed that the scheme reduces to a three points, first order scheme at extrema.

The rest of this section is devoted to comments upon the proof of our main result that any generalized monotone scheme converges to the entropy solution of (1.1)(1.2) provided (1.3)-(1.4) holds. Our approach was driven by Yang's paper [38] on semi-discrete schemes. However, our technical arguments differ substantially from the ones in [38]. In particular we restrict attention to initial data having a locally finite number of extrema, making the tracking of paths of extremum values almost a trivial matter. The main part of our proof is studying the convergence of the traces of the approximate solution along it. We make use of the quadratic decay property above to exclude the formation of a "cusp" near extremum values, which could lead to entropy-violating shock. This is the main contribution of this present paper. We do not believe that the extension of our proof to arbitrary BV data is straightforward. The result we obtain seems satisfactory, however, since condition (1.4) covers "generic" initial data.

The proof distinguishes between the non-increasing parts and non-decreasing parts of the solution, and is based on several observations, as follows. We use the notation $u^{h}$ for the approximate solutions, $v$ for the limiting solution, and $h$ for the mesh size.

First of all, the strong maximum principle ensures that the $u^{h}$ 's are total variation diminishing in time, so of uniformly bounded total variation. The strong maximum principle makes it easy for us to define a discrete path, and with each time step the discrete path moves at most one grid point. As a consequence, the discrete path is Lipschitz continuous. (This is a major difference between the present paper and Yang's paper, in which the construction of the path and the limiting paths is much more involved.)

By Helly's Theorem, the scheme converges in the strong $L^{1}$ topology to a limiting function, say $v$, which according to Lax-Wendroff's theorem is a weak solution to (1.1)-(1.2). It remains to prove that $v$ is the entropy solution. We show that the strong maximum principle in fact implies a discrete analogue of the monotonicity property. Relying on (1.4), we construct a (locally) finite family of Lipschitz 
continuous paths in the plane by tracking the local extrema in $u^{h}$. The paths are shown to converge in the uniform topology to limiting curves.

Next we make the following two observations. On one hand, in a non-increasing region for $u^{h}$, the function $v$ is also non-increasing, and so can only admit nonincreasing jumps. Thus $v$ satisfies the Lax shock admissibility inequality (1.6) in the non-increasing regions. On the other hand, a discrete cell entropy inequality, by assumption, holds in the non-decreasing regions of $u^{h}$. So $v$ satisfies (1.5) in the non-decreasing regions.

It remains to prove that $v$ has only non-increasing jumps along any path of extrema. This is the most interesting part of the proof. Let $\psi^{h}$ be an approximate path of extrema, and let $\psi$ be its uniform limit. The path $\psi$ is the boundary separating two regions where the analysis in the paragraph above applies. Indeed, (1.5) holds in the side where $v$ is non-increasing and (1.6) holds in the side where $v$ is non-decreasing. A specific proof must be provided to determine the behavior of $v$ along the path. We analyze the entropy production in a small region of the plane limited on one side by the path $\psi^{h}$. In the course of this proof we derive a uniform bound for the time integral of the local oscillation in space along the path $\psi^{h}$, which is a direct consequence of the quadratic decay property mentioned above. For the sake of simplicity, we use here the assumption that the scheme reduces to a three points, first order scheme at extrema.

Note that assumption (1.3) is not used in the construction of the extremum paths, but is essential in the convergence analysis which strongly relies upon (1.6), only valid for convex fluxes.

Our analysis shows that, for a class of difference schemes, certain approximate generalized characteristics - those issuing from an extremum point of the initial data - can be constructed for scalar conservation laws with convex flux. Constructing approximate generalized characteristics issuing from an arbitrary point remains a challenging open problem. Recall that, for the random choice scheme, Glimm-Lax [10] did obtain a general theory of approximate generalized characteristics (applicable to systems, as well).

The organization of this paper is as follows. In Section 2, we define the class of generalized monotone schemes and state the main result of convergence, Theorem 2.3. Section 3 contains the proof of the main result. In Section 4, we apply Theorem 2.3 to the MUSCL scheme and provide some additional remarks.

\section{Generalized monotone schemes}

This section introduces a class of TVD schemes which are built to closely mimic an essential property of the entropy solutions to (1.1), i.e. the monotonicity property: the number of local extrema is a non-increasing function of time, and local maximum/minimum values decrease/increase monotonically in time. See the Appendix for a precise statement. Here we investigate this property from a numerical standpoint. Monotone and TVD schemes actually do not necessarily satisfy this property (see Section 4 for an example), and a more restricted class, the generalized monotone schemes, is natural.

We consider a $(2 k+1)$-point difference scheme in conservation form for the approximation of (1.1)-(1.2):

$$
u_{j}^{n+1}=u_{j}^{n}-\lambda\left(g_{j+1 / 2}^{n}-g_{j-1 / 2}^{n}\right), \quad n \geq 0, j \in \mathbf{Z},
$$


where we use the notation $g_{j+1 / 2}^{n}=g\left(u_{j-k+1}^{n}, \ldots, u_{j+k}^{n}\right)$, and $\lambda=\tau / h$ is the ratio of the time-increment $\tau$ by the space-increment $h$. We set $t_{n}=n \tau, x_{j}=j h$, and $x_{j+1 / 2}=(j+1 / 2) h$. The value $u_{j}^{n}$ presumably is an approximation of the exact solution at the point $\left(x_{j}, t_{n}\right)$. As usual, the numerical flux $g: \mathbb{R}^{2 k} \rightarrow \mathbb{R}$ is assumed to be locally Lipschitz continuous and consistent with $f$, i.e. $g(v, \ldots, v)=f(v)$ for all $v$. Note that $g$ may depend on $\lambda$. For definiteness, we set

$$
u_{j}^{0}=\frac{1}{h} \int_{x_{j-1 / 2}}^{x_{j+1 / 2}} u_{0}(y) d y .
$$

This is sufficient for second order accuracy. For higher orders, one should use a Runge-Kutta time-step method (Shu [34]). We also define the piecewise constant function $u^{h}: \mathbb{R} \times \mathbb{R}_{+} \rightarrow \mathbb{R}$ by

$$
u^{h}(x, t)=u_{j}^{n}, \quad t_{n} \leq t<t_{n+1}, x_{j-1 / 2} \leq x<x_{j+1 / 2} .
$$

By construction $u^{h}$ is a right continuous function. For simplicity we assume the following CFL restriction:

$$
\lambda \sup _{v}\left|f^{\prime}(v)\right| \leq 1 / 4
$$

Several of the properties below would still hold if, in (2.4), one replaces $1 / 4$ by 1 , although the proofs then become less clear geometrically.

The (first order) Godunov scheme is based on exact solutions to (1.1), and is a good prototypical example to lead us to defining a class of (high order) schemes consistent with the monotonicity property.

A main ingredient is the Riemann problem. Given two states $v$ and $w$, we define $R(. ; v, w)$ to be the entropy solution to (1.1)-(1.2) with, here,

$$
u_{0}(x)=v \quad \text { if } x<0, \quad u_{0}(x)=w \quad \text { if } x>0 .
$$

As is well-known, $R(\cdot ; v, w)$ depends on the self similarity variable $x / t$ only, and is given by a closed formula. If $v \leq w, R$ is a rarefaction wave; if $v>w$, a shock wave. More important,

$$
R\left(\frac{x}{t} ; v, w\right) \text { is a monotone function connecting } v \text { to } w \text {. }
$$

In the Godunov scheme, one solves Riemann problems and, at each time level, one projects the solution on the space of piecewise constant functions. If $u^{h}\left(t_{n}\right)$ is known, let $\tilde{u}(x, t)$ for $t \geq t_{n}$ be the entropy solution to (1.1) assuming the Cauchy data

$$
\tilde{u}\left(t_{n}+\right)=u^{h}\left(t_{n}+\right)
$$

Since $u^{h}\left(t_{n}+\right)$ is a piecewise constant function, $\tilde{u}$ is obtained explicitly by gluing together the Riemann solutions $R\left(. ; u_{j}^{n}, u_{j+1}^{n}\right)$. In view of $(2.4)$, there is no interaction between two nearby solutions, at least for $t \in\left(t_{n}, t_{n+1}\right)$. Set

$$
u^{h}\left(x, t_{n+1}+\right)=\frac{1}{h} \int_{x_{j-1 / 2}}^{x_{j+1 / 2}} \tilde{u}\left(y, t_{n+1}\right) d y, \quad x_{j-1 / 2} \leq x<x_{j+1 / 2} .
$$

Using the conservative form of (1.1), the scheme can be written in the form (2.1) with $k=1$ and $g=g_{G}$ given by

$$
g_{G}(v, w)=f(R(0+; v, w)) \quad \text { for all } v \text { and } w .
$$


The proofs of (2.7)-(2.10) stated below are classical; e.g. [3], [6], [16], [26]. On one hand, one can think of the Godunov scheme geometrically as a two-step method: a marching step based on exact (Riemann) solutions and an $L^{2}$ projection step. In view of (2.5) and the monotonicity property of the $L^{2}$ projection, one can easily get a simple geometrical proof of the properties listed below. On the other hand, an algebraic approach is based on the explicit formula deduced from (2.6).

The Godunov scheme is monotone: the function $g_{G}$ is non-decreasing with respect to its first argument, and non-increasing with respect to its second. This property implies that the scheme is monotonicity preserving, i.e.,

$$
\begin{aligned}
& \text { if } u_{j_{1}}^{n}, u_{j_{1}+1}^{n}, \ldots, u_{j_{2}}^{n} \text { is a non-increasing (resp. non-decreasing) sequence } \\
& \text { for some indices } j_{1}<j_{2} \text {, so is the sequence } u_{j_{1}+1}^{n+1}, u_{j_{1}+2}^{n+1}, \ldots, u_{j_{2}-1}^{n+1} \text {. }
\end{aligned}
$$

The Godunov scheme satisfies the local maximum principle, i.e.,

$$
\min \left(u_{j-1}^{n}, u_{j}^{n}, u_{j+1}^{n}\right) \leq u_{j}^{n+1} \leq \max \left(u_{j-1}^{n}, u_{j}^{n}, u_{j+1}^{n}\right)
$$

for all $n \geq 0$ and $j \in \mathbf{Z}$. In fact (2.7) and (2.8) are shared by both steps in the Godunov scheme. It will be convenient for us to rewrite (2.8) in term of the jumps of $u^{h}$ at the endpoints of a cell:

$$
\min \left(u_{j+1}^{n}-u_{j}^{n}, u_{j-1}^{n}-u_{j}^{n}, 0\right) \leq u_{j}^{n+1}-u_{j}^{n} \leq \max \left(u_{j+1}^{n}-u_{j}^{n}, u_{j-1}^{n}-u_{j}^{n}, 0\right) .
$$

Any monotone scheme -in particular the Godunov scheme- satisfies a discrete analogue of (1.5) for every convex entropy pair $(U, F)$ :

$$
U\left(u_{j}^{n+1}\right)-U\left(u_{j}^{n}\right)-\lambda\left(G_{j+1 / 2}^{n}-G_{j-1 / 2}^{n}\right) \leq 0, \quad n \geq 0, j \in \mathbf{Z} .
$$

In (2.9), $G_{j+1 / 2}^{n}=G\left(u_{j-k+1}^{n}, \ldots, u_{j+k}^{n}\right)$, and $G$ is a numerical entropy flux consistent with $F$; that is, $G(v, v \ldots, v)=F(v)$ for all $v$.

Finally, concerning the local behavior of $u^{h}$ in the neighborhood of local extrema, it is known that, say for local maximum,

$$
u_{j}^{n+1} \leq u_{j}^{n} .
$$

A similar property holds for local minima.

In fact the classical properties (2.8) and (2.10) can be improved as follows.

Proposition 2.1. Under assumptions (1.3) and (2.4), the Godunov scheme satisfies the following two properties:

1. The strong local maximum principle:

$$
\frac{1}{2} \min \left(u_{j+1}^{n}-u_{j}^{n}, u_{j-1}^{n}-u_{j}^{n}, 0\right) \leq u_{j}^{n+1}-u_{j}^{n} \leq \frac{1}{2} \max \left(u_{j+1}^{n}-u_{j}^{n}, u_{j-1}^{n}-u_{j}^{n}, 0\right) .
$$

2. The quadratic decay property at local extrema, that is, e.g. for a maximum,

$$
\text { if } u_{j}^{n} \text { is a local maximum value, } u_{j}^{n+1} \leq u_{j}^{n}-\alpha \min _{ \pm}\left(\left(u_{j}^{n}-u_{j \pm 1}^{n}\right)^{2}\right) \text {, }
$$

with $\alpha=\lambda \inf f^{\prime \prime} / 2$.

The proof of (2.11) is straightforward from a geometrical standpoint. If also follows from Proposition 4.1, to be established later in Section 4. Note that the coefficient $1 / 4$ in (2.4) is essential for (2.11) to hold. Observe that (2.11) is stronger than (2.8)- $\left(2.8^{\prime}\right)$ and controls the time-increment (i.e., $u_{j}^{n+1}-u_{j}^{n}$ ) of the solution in the cell $j$ in term of the jumps at the endpoints: the values $u_{j}^{n}$ evolves "slowly" 
as $t_{n}$ increases. As we shall see, this property implies that the scheme satisfies a discrete analogue of the monotonicity property.

Estimate (2.12) is stronger than (2.10) and shows that the decrease/increase of a maximum/minimum is controlled by the quadratic oscillation of $u^{h}$ near this extremum. It is a truly nonlinear property of the Godunov flux. It will be used below to prove that a cusp cannot form near extremum points. For convenience, the proof of (2.12) is postponed to Section 4, where second-order approximations are treated as well.

In [12], Goodman and LeVeque derive for the Godunov method a discrete version of the Oleinik entropy inequality. In particular, this shows that the Godunov scheme spreads rarefaction waves at the correct rate. Our estimate (2.12) is, at least in spririt, similar to this spreading estimate, and expresses the spreading of extremum values.

We are now ready to introduce a class of high-order schemes based on the properties derived in Proposition 2.1.

Definition 2.2. The scheme (2.1) is said to be a generalized monotone scheme if any sequence $\left\{u_{j}^{n}\right\}$ generated by (2.1) satisfies the following three conditions:

(1) the strong local maximum principle (2.11),

(2) the cell entropy inequality (2.9) for one strictly convex pair $(U, F)$ in any non-decreasing region, including local extrema,

(3) the quadratic decay property at local extrema (2.12) for some constant $\alpha>0$.

It is also assumed that the numerical flux and the numerical entropy flux are essentially two-point functions at local extrema.

According to Proposition 2.1, the (first order) Godunov scheme belongs to the class described in Definition 2.2. Section 4 will show that there exist high order accurate schemes satisfying the conditions in Definition 2.2. Our main convergence result is:

Theorem 2.3. Let (2.1) be a generalized monotone scheme. Assume that assumptions (1.3)-(1.4) hold together with (2.4). Then the scheme (2.1)

(1) is $L^{\infty}$ stable, i.e.,

$$
\inf _{l \in \mathbf{Z}} u_{l}^{n} \leq u_{j}^{n+1} \leq \sup _{l \in \mathbf{Z}} u_{l}^{n}, \quad n \geq 0, j \in \mathbf{Z},
$$

(2) is total variation diminishing, i.e.,

$$
\sum_{j \in \mathbf{Z}}\left|u_{j+1}^{n+1}-u_{j}^{n+1}\right| \leq \sum_{j \in \mathbf{Z}}\left|u_{j+1}^{n}-u_{j}^{n}\right|, \quad n \geq 0,
$$

(3) and converges in the $L_{\text {loc }}^{p}$ strong topology for all $p \in[1, \infty)$ to the entropy solution of (1.1)-(1.2).

The proof of Theorem 2.3 is given in Section 3.

Theorem 2.3 is satisfactory from a practical standpoint. Suppose that $u_{0}$ is an arbitrary BV function, and we wish to compute an approximation to the solution $u$ of (1.1)-(1.2) of order $\varepsilon>0$ in the $L^{1}$ norm. Let us determine first an approximation of $u_{0}$, say $u_{0, \varepsilon}$, that has a finite number of local extrema and is such that

$$
\left\|u_{0, \varepsilon}-u_{0}\right\|_{L^{1}(\mathbb{R})} \leq \varepsilon
$$


Applying a generalized monotone scheme to the initial condition $u_{0, \varepsilon}$ yields an approximate solution $u_{\varepsilon}^{h}$ that, in view of Theorem 2.3, satisfies

$$
\left\|u_{\varepsilon}^{h}-u_{\varepsilon}\right\|_{L^{1}(\mathbb{R})} \leq o(h) \leq \varepsilon
$$

for $h$ is small enough, where $u_{\varepsilon}$ is the entropy solution associated with the initial condition $u_{0, \varepsilon}$. Since the semigroup of solutions associated with (1.1) satisfies the $L^{1}$ contraction property, one has

$$
\left\|u_{\varepsilon}-u\right\|_{L^{1}(\mathbb{R})} \leq\left\|u_{0, \varepsilon}-u_{0}\right\|_{L^{1}(\mathbb{R})} \leq \varepsilon,
$$

and therefore

$$
\begin{aligned}
\left\|u_{\varepsilon}^{h}-u\right\|_{L^{1}(\mathbb{R})} & \leq\left\|u_{\varepsilon}^{h}-u_{\varepsilon}\right\|_{L^{1}(\mathbb{R})}+\left\|u_{\varepsilon}-u\right\|_{L^{1}(\mathbb{R})} \\
& \leq 2 \varepsilon .
\end{aligned}
$$

\section{Convergence Analysis}

The proof of Theorem 2.3 is decomposed into several lemmas, Lemmas 3.1-3.11. For the whole of this section, we assume that the hypotheses of Theorem 2.3 are satisfied.

We introduce first some notation and terminology. We call $u_{j}^{n}$ a local maximum or a local minimum if there exist two indices $j_{*}$ and $j^{*}$ with $j_{*} \leq j \leq j^{*}$ such that

$$
u_{j_{*}}^{n}=u_{j_{*}+1}^{n}=\cdots=u_{j^{*}}^{n}>\max \left(u_{j_{*}-1}^{n}, u_{j^{*}+1}^{n}\right)
$$

or

$$
u_{j_{*}}^{n}=u_{j_{*}+1}^{n}=\cdots=u_{j^{*}}^{n}<\min \left(u_{j_{*}-1}^{n}, u_{j^{*}}^{n}\right) .
$$

In such a case, there is no need to distinguish between the extrema $u_{j_{*}}^{n}, u_{j_{*}+1}^{n}$, $\ldots, u_{j^{*}}^{n}$. Based on the strong maximum principle (2.11), we show in Lemmas 3.1 and 3.2 that the scheme satisfies a discrete form of the monotonicity property. We construct a family of paths in the $(x, t)$-plane by tracing in time the points where the approximate solution $u^{h}(t)$ achieves its local extremum values. One difficulty is proving that the interaction of two (or more) paths does not create new paths, so the total number of paths at any given time remains less than or equal to the initial number of local extrema in $u_{0}$. In passing we observe that an extremum point moves one grid point at each time-step, at most.

Lemma 3.1. For some $j_{*}<j^{*}$, suppose that the sequences $u_{j_{*}-3}^{n}, u_{j_{*}-2}^{n}, u_{j_{*}-1}^{n}$, $u_{j_{*}}^{n}$ and $u_{j^{*}}^{n}, u_{j^{*}+1}^{n}, u_{j^{*}+2}^{n}, u_{j^{*}+3}^{n}$ are two monotone sequences, no specific assumption being made on the values $u_{j}^{n}, j_{*} \leq j \leq j^{*}$. Then the number $\nu^{\prime}$ of extrema in the sequence

$$
S_{n+1}:=\left(u_{j}^{n+1}\right)_{j_{*}-2 \leq j \leq j^{*}+2}
$$

is less than or equal to the number $\nu$ of extrema in

$$
S_{n}:=\left(u_{j}^{n}\right)_{j_{*}-2 \leq j \leq j^{*}+2} .
$$

When $\nu^{\prime} \geq 1$, there exists a one-to-one correspondence between $\nu^{\prime}$ local extrema of $S_{n}$ and the $\nu^{\prime}$ local extrema of $S_{n+1}$ with the following property: if a maximum/minimum $u_{j}^{n}$ is associated with a maximum/minimum $u_{j^{\prime}}^{n+1}$, then

$$
\left|j^{\prime}-j\right| \leq 1 \quad \text { and } \quad u_{j^{\prime}}^{n+1} \leq u_{j}^{n} \text {, resp. } u_{j^{\prime}}^{n+1} \geq u_{j}^{n} .
$$


Proof. We distinguish between various cases depending on the number of local extrema in the sequence $S_{n}$ and construct the one-to-one correspondence.

If $S_{n}$ has no local extremum, for instance is non-decreasing, then $S_{n+1}$ is also non-decreasing. This indeed follows from the inequalities (2.11), which reduce in this case to

$$
\cdots \leq \frac{1}{2}\left(u_{j-1}^{n}+u_{j}^{n}\right) \leq u_{j}^{n+1} \leq \frac{1}{2}\left(u_{j+1}^{n}+u_{j}^{n}\right) \leq u_{j+1}^{n+1} \leq \cdots .
$$

Consider next the case that $S_{n}$ has exactly one local extremum, say a local maximum at some $u_{l}^{n}$. The same argument as above shows that the sequences $\left\{u_{j}^{n+1}\right\}_{j_{*} \leq j \leq l-1}$ and $\left\{u_{j}^{n+1}\right\}_{l+1 \leq j \leq j^{*}}$ are non-decreasing and non-increasing respectively. Therefore we only need to exclude the case that both $u_{l-1}^{n+1}>u_{l}^{n+1}$ and $u_{l+1}^{n+1}>u_{l}^{n+1}$, which would violate the monotonicity property since $S_{n+1}$ in this case would have two local maximum and one local minimum, so two new extrema. Indeed assume that the latter would hold; then using (2.11) at the points $l-1, l$, and $l+1$ gives us

$$
\begin{gathered}
u_{l-1}^{n+1} \leq \frac{1}{2}\left(u_{l-1}^{n}+u_{l}^{n}\right), \\
u_{l}^{n}+\frac{1}{2} \min \left(u_{l \pm 1}^{n}-u_{l}^{n}\right) \leq u_{l}^{n+1},
\end{gathered}
$$

and

$$
u_{l+1}^{n+1} \leq \frac{1}{2}\left(u_{l+1}^{n}+u_{l}^{n}\right),
$$

which are incompatible with the inequalities $u_{l-1}^{n+1}>u_{l}^{n+1}$ and $u_{l+1}^{n+1}>u_{l}^{n+1}$.

Consider now the case that $S_{n}$ has two local extrema, say one local maximum at $l$ and one local minimum at $m$ with $l<m$. We distinguish between three cases:

If $l<m-2$, then the two extrema cannot "interact" and the previous arguments show that the solution at time $t_{n+1}$ has the same properties.

If $l=m-2$, the two extrema can interact. Using (2.11) at each point $j=$ $l-1, \cdots, l+3$, one gets

$$
\begin{gathered}
u_{l-1}^{n+1} \leq \frac{1}{2}\left(u_{l-1}^{n}+u_{l}^{n}\right), \\
u_{l}^{n}+\frac{1}{2} \min \left(u_{l \pm 1}^{n}-u_{l}^{n}\right) \leq u_{l}^{n+1},
\end{gathered}
$$

and

$$
u_{l+1}^{n+1} \leq \frac{1}{2}\left(u_{l+1}^{n}+u_{l}^{n}\right)
$$

and also

$$
\begin{gathered}
u_{l+1}^{n+1} \leq \frac{1}{2}\left(u_{l+1}^{n}+u_{l+2}^{n}\right), \\
u_{l+2}^{n}+\frac{1}{2} \min \left(u_{l+2 \pm 1}^{n}-u_{l+2}^{n}\right) \leq u_{l+2}^{n+1},
\end{gathered}
$$

and

$$
u_{l+3}^{n+1} \leq \frac{1}{2}\left(u_{l+3}^{n}+u_{l+2}^{n}\right) .
$$

It is not hard to see that these inequalities imply that $S_{n+1}$ : 
(1) either has one maximum at $j=l-1, l$, or $l+1$ and a minimum at $j=l+1, l+2$, or $l+3$;

(2) or is non-decreasing.

In the first case, we achieve the property we wanted. In the second case, there is no extremum at the time $t_{n+1}$.

This analysis can be extended to the case that several extrema can "interact"; we omit the details. Property (3.1) is a consequence of condition (2.4): an extremum point can only move up to one grid point at each time step.

Consider the initial condition $u_{0}$ and its approximation $u^{h}(0)$ defined by $L^{2}$ projection, cf. (2.2). Locate the minimum and maximum values in the initial data $u_{0}$. For $h$ much smaller than the minimal distance between two extrema, $u^{h}(0)$ has the same number of extrema as $u_{0}$ and the same increasing/decreasing behavior as $u_{0}$. Indeed, there exist indices $J_{q}^{h}(0)$ for $q$ in a set of consecutive integers $E\left(u_{0}\right)$ depending on $u_{0}$ but not on $h$, such that

$$
\begin{aligned}
& u_{j}^{0} \text { is non-decreasing for } J_{2 p}^{h}(0) \leq j \leq J_{2 p+1}^{h}(0), \\
& u_{j}^{0} \text { is non-increasing for } J_{2 p-1}^{h}(0) \leq j \leq J_{2 p}^{h}(0) .
\end{aligned}
$$

Those indices are not uniquely determined in the case that $u_{0}$ is constant on an interval associated with a local extremum. Since $u_{0}$ has a locally finite number of local extrema, there exists a partition of $\mathbf{Z}$ into intervals $\left(j_{*}, j^{*}\right)$ in which the hypothesis of Lemma 3.1 holds. It is an easy matter to use the one-to-one correspondence in Lemma 3.1 and trace forward in time up to time $t_{1}=\tau$ the locations of the extrema in $\left(j_{*}, j^{*}\right)$. At each time level a (possibly new) partition of $\mathbf{Z}$ is considered and Lemma 3.1 is used again. Indeed, the values $J_{q}^{h}(n+1)$ in Lemma 3.2 below are defined from the $J_{q}^{h}(n)$ 's according to the one-to-one correspondence established in Lemma 3.1. Finally, piecewise affine and continuous paths are obtained by connecting together the points of local extrema. It may happen that the number of extrema decreases from time $t_{n}$ to $t_{n+1}$. In such a case, one path, at least, can no longer be further extended in time and so, for that purpose, we introduce a "stopping time", denoted by $T_{q}^{h}=t_{n}$.

The following lemma is established.

Lemma 3.2. There exist continuous and piecewise affine curves $\psi_{q}^{h}:\left[0, T_{q}^{h}\right] \rightarrow \mathbb{R}$ for $q \in E\left(u_{0}\right)$, passing through the mesh points $\left(x_{J_{q}^{h}(n)}, t_{n}\right)$ and having the following properties:

$$
\psi_{q}^{h}(t)=x_{J_{q}^{h}(n)}+\frac{t-t_{n}}{\tau}\left(x_{J_{q}^{h}(n+1)}-x_{J_{q}^{h}(n)}\right), \quad t \in\left[t_{n}, t_{n+1}\right],
$$

for each $n=0,1,2, \ldots, N_{q}^{h}$ with $T_{q}^{h}=N_{q}^{h} \tau \leq \infty$,

there are only a finite number (uniformly bounded w.r.t. $h$ )

of curves $\psi_{q}^{h}$ on each compact set

and

$$
\begin{aligned}
& x \in\left(\psi_{2 p}^{h}\left(t_{n}\right), \psi_{2 p+1}^{h}\left(t_{n}\right)\right) \mapsto u^{h}\left(x, t_{n}\right) \quad \text { is non-decreasing, } \\
& x \in\left(\psi_{2 p-1}^{h}\left(t_{n}\right), \psi_{2 p}^{h}\left(t_{n}\right)\right) \mapsto u^{h}\left(x, t_{n}\right) \text { is non-increasing. }
\end{aligned}
$$


Furthermore, the functions $w_{q}^{h}:\left[0, T_{q}^{h}\right] \rightarrow \mathbb{R}$ defined by

$$
w_{q}^{h}(t)=u_{J_{q}^{h}(n)}^{n} \quad \text { for } \quad t_{n} \leq t<t_{n+1}
$$

are non-decreasing if $q$ is even, and non-increasing if $q$ is odd.

Remark 3.3. 1) The definition (3.3) is not essential. All the results below still hold if $\psi_{q}^{h}$ is replaced by any (uniformly) Lipschitz continuous curve passing through the mesh points $\left(x_{J_{q}^{h}(n)}, t_{n}\right)$. As a matter of fact, it is an open problem to show the strong convergence of the derivatives of approximate paths. By comparison, for the approximate solutions built by the random choice scheme, Glimm and Lax [10] proved the a.e. convergence of the first order derivatives of the paths.

2) Introducing the stopping times $T_{q}^{h}$ is necessary. At those times, certain paths cross each other and their extension in time is not well-defined. For instance, a path of maximum and a path of minimum can cross and "cancel out". The case of exact solutions (cf. the appendix) is simpler in this respect: the paths can be defined to be characteristic curves for all times, even when they are no longer paths of extrema.

3 ) It is not interesting to trace the minimal (or maximal) paths of extrema in the approximate solution. Such paths would not converge to the paths obtained in the continuous case.

By construction, cf. (3.4), a path "jumps" up to one grid point at each time-step. So the slope of a path remains uniformly bounded by $1 / \lambda$ and the curves $\psi_{q}^{h}$ are bounded in the $W_{\text {loc }}^{1, \infty}$ norm, uniformly with respect to $h$ and $q$. On the other hand, Lemma 3.3 implies that the scheme is TVD, so $T V\left(u^{h}\left(t_{n}\right)\right)$ is uniformly bounded. We thus conclude that the approximate solutions and the paths of extrema are strongly convergent, as stated in the following Lemmas 3.4 and 3.5. For simplicity, we keep the same notation for a sequence and a subsequence.

Lemma 3.4. There exist times $T_{q} \in[0,+\infty]$ and Lipschitz continuous curves $\psi_{q}$ : $\left(0, T_{q}\right) \rightarrow \mathbb{R}$ such that

$$
T_{q}^{h} \rightarrow T_{q} \quad \text { as } h \rightarrow 0,
$$

and

$$
\psi_{q}^{h} \rightarrow \psi_{q} \quad \text { uniformly on each compact subset of } C^{0}\left(\left[0, T_{q}\right)\right) .
$$

Lemma 3.5. The sequence $u^{h}$ satisfies estimates (2.13)-(2.14), and so is uniformly stable in the $L^{\infty}([0, \infty), B V(\mathbb{R}))$ and Lip $\left([0, \infty), L^{1}(\mathbb{R})\right)$ norms. There exists a function $v$ in the same spaces such that

$$
u^{h}(x, t) \rightarrow v(x, t) \quad \text { for all times } t \geq 0 \text { and almost every } x \in \mathbb{R},
$$

and there exist functions $w_{q}$ in $B V\left(\left(0, T_{q}\right), \mathbb{R}\right)$ such that

$$
w_{q}^{h} \rightarrow w_{q} \quad \text { almost everywhere on }\left(0, T_{q}\right)
$$

for all $q \in E\left(u_{0}\right)$.

The convergence results (3.10) and (3.11) hold in particular at each point of continuity of $v(t)$ and $w_{q}$, respectively. Introduce now the following three sets, which provide us with a partition of the $(x, t)$-plane into increasing/decreasing 
regions for $v$ :

$$
\begin{aligned}
& \Omega_{1}(v)=\left\{(x, t) \mid \psi_{2 p_{1}}(t)<x<\psi_{2 p_{2}+1}(t), p_{1} \leq p_{2}, t<T_{2 p_{1}}, t<T_{2 p_{2}+1},\right. \\
& \text { and } \left.t>T_{q} \text {, for all } q=2 p_{1}+1, \ldots, 2 p_{2}\right\}, \\
& \Omega_{2}(v)=\left\{(x, t) \mid \psi_{2 p_{1}-1}(t)<x<\psi_{2 p_{2}}(t), p_{1} \leq p_{2}, t<T_{2 p_{1}-1}, t<T_{2 p_{2}},\right. \\
& \text { and } \left.t>T_{q} \text {, for all } q=2 p_{1}, \ldots, 2 p_{2}-1\right\} \text {, } \\
& \Omega_{3}(v)=\text { Closure }\left\{\left(\psi_{q}(t), t\right) \text {, for all relevant values of } t \text { and } q\right\} \text {. }
\end{aligned}
$$

The set $\Omega_{3}(v)$, by construction, contains all of the curves $\psi_{q}$ including their end points. The sets $\Omega_{1}(v)$ and $\Omega_{2}(v)$ are open and contain regions limited by curves in $\Omega_{3}(v)$. These definitions take into account the fact that the path need not be defined for all times. Observe also that an arbitrary point in $\Omega_{3}(v)$ need not be a point of extremum value for $v$. The decomposition under consideration is not quite the obvious partition of the $(x, t)$-plane into regions of monotonicity for $v$. Strictly speaking, the sets $\Omega_{j}(v)$ may not be determined from the function $v$ alone.

Using Lemmas 3.2, 3.4, and 3.5, we immediately check that:

Lemma 3.6. The limiting functions satisfy the properties:

$$
\begin{aligned}
& v_{/ \Omega_{1}(v)}(t) \text { is non-decreasing in each subcomponent of } \Omega_{1}(v), \\
& v_{/ \Omega_{2}(v)}(t) \text { is non-increasing in each subcomponent of } \Omega_{2}(v),
\end{aligned}
$$

and

$$
w_{q} \text { is non-decreasing if } q \text { is even and non-increasing if } q \text { is odd. }
$$

Since the scheme is consistent, conservative, and converges in the $L^{1}$ strong norm, we can pass to the limit in (2.1). It follows that $v$ is a weak solution to (1.1). We note that, in the set $\Omega_{1}(v)$, the functions $u^{h}$, and also $v$, are non-increasing. The Lax shock inequality holds for both $u^{h}$ and $v$. On the other hand, the cell entropy inequality (2.9) holds for $u^{h}$ in the non-decreasing regions, i.e., in $\Omega_{2}(v)$. The passage to the limit in (2.9) is a classical matter.

Lemma 3.7. The function $v$ is a weak solution to equation (1.1) and satisfies

$$
v(x-, t) \geq v(x+, t) \quad \text { in the set } \Omega_{1}(v)
$$

and

$$
\partial_{t} U(v)+\partial_{x} F(v) \leq 0 \quad \text { in the set } \Omega_{2}(v) .
$$

The rest of this section is devoted to proving that the Lax shock inequality holds along the paths $\psi_{q}$ which we will attain in Lemma 3.10. In a first stage, we prove:

Lemma 3.8. Along each path of extremum $\psi_{q}$ and for almost every $t \in\left(0, T_{q}\right)$, one of the following holds:

$$
w_{q}(t)=v\left(\psi_{q}(t)-, t\right) \quad \text { or } \quad w_{q}(t)=v\left(\psi_{q}(t)+, t\right) .
$$

Roughly speaking, (3.16) means that that no cusp-like layer can form in the scheme near local extrema. The idea of the proof of Lemma 3.8 is as follows: we are going to integrate the discrete form of the conservation law (2.1) on a (small) domain limited on one side by an approximate path of extremum; then we shall integrate by parts and pass to the limit as $h \rightarrow 0$. Finally, we shall let the domain shrink and reduce to the path itself. To determine the limits of the relevant boundary terms as $h \rightarrow 0$, we have to justify the passage to the limit in particular 
in the numerical fluxes evaluated along the approximate path. Lemma 3.9 below provides us with an a priori estimate for the oscillation of $u^{h}$ along the path, which follows from the quadratic decay property (2.12).

Lemma 3.9. Along a path of extremum values $\psi_{q}^{h}$, we have

$$
\begin{aligned}
& \beta \sum_{n=n_{-}}^{n=n_{+}} \min \left(\left|u_{J_{q}^{h}(n) \pm 1}^{n}-u_{J_{q}^{h}(n)}^{n}\right|,\left|u_{J_{q}^{h}(n) \pm 1}^{n}-u_{J_{q}^{h}(n)}^{n}\right|^{2}\right) \\
& \quad \leq u_{J_{q}^{h}\left(n_{-}\right)}^{n_{-}}-u_{J_{q}^{h}\left(n_{+}\right)+1}^{n_{+}},
\end{aligned}
$$

for all $0 \leq n_{-} \leq n_{+} \leq N_{q}^{h}$, where

$$
\beta=\min (\alpha, 1 / 2) .
$$

Proof of Lemma 3.8. We will prove that, for almost every $t$ in $\left(0, T_{q}\right)$, the following three Rankine-Hugoniot like relations hold:

$$
\begin{gathered}
-\frac{d \psi_{q}}{d t}(t)\left(v\left(\psi_{q}(t)+, t\right)-v\left(\psi_{q}(t)-, t\right)\right) \\
+f\left(v\left(\psi_{q}(t)+\right), t\right)-f\left(v\left(\psi_{q}(t)-, t\right)\right)=0 \\
-\frac{d \psi_{q}}{d t}(t)\left(v\left(\psi_{q}(t) \pm, t\right)-w_{q}(t)\right)+f\left(v\left(\psi_{q}(t) \pm, t\right)\right)-f\left(w_{q}(t)\right)=0 .
\end{gathered}
$$

Since there is only one non-trivial pair of values that achieves a Rankine-Hugoniot relation for a scalar conservation law with a strictly convex flux and a given shock speed $d \psi_{q}(t) / d t$, the desired conclusion (3.16) follows immediately from (3.19)(3.20).

Observe that (3.19) is nothing but the standard Rankine-Hugoniot relation, since $v$ is a weak solution to (1.1) and $\psi_{q}$ is Lipschitz continuous. For definiteness we prove (3.20) in the case of the "+" sign. The proof of (3.20) with "-" sign is entirely similar. (Actually only one of the two relations in (3.20) suffice for the present proof.)

Let $\theta(x, t)$ be a test-function having its support included in a neighborhood of the curve $\psi_{q}$ and included in the strip $\mathbb{R} \times\left(0, T_{q}\right)$. So for $h$ small enough the support of $\theta$ is included in $\mathbb{R} \times\left(0, T_{q}^{h}\right)$, and all the quantities to be considered below make sense. Let us set $\theta_{j}^{n}=\theta\left(x_{j}, t_{n}\right)$. To make use of estimate (3.17), it is necessary to define a "shifted" path $\tilde{\psi}_{q}^{h}$, to be used instead of $\psi_{q}^{h}$. So we consider the set of indices

$$
\mathcal{P}_{q}^{h}=\left\{(j, n) \mid j \geq J_{q}^{h}(n)+\epsilon_{q}^{h}(n)\right\},
$$

where $\epsilon_{q}^{h}(n)=0$ (respectively $\left.\epsilon_{q}^{h}(n)=1\right)$ if $J_{q}^{h}(n)-1$ (resp. $\left.J_{q}^{h}(n)+1\right)$ achieves the minimum in the left hand side of (3.17). A shifted path is defined by

$$
\tilde{\psi}_{q}^{h}(t)=x_{J_{q}^{h}(n)+\epsilon_{q}^{h}(n)}+\frac{t-t_{n}}{\tau}\left(x_{J_{q}^{h}(n+1)+\epsilon_{q}^{h}(n+1)}-x_{J_{q}^{h}(n)+\epsilon_{q}^{h}(n)}\right), \quad t \in\left[t_{n}, t_{n+1}\right] .
$$

Introducing the shifts $\epsilon_{q}^{h}(n)$ does not modify the convergence properties of the path. It is not hard to see, using solely the fact that the path is uniformly bounded in Lipschitz norm, that as $h \rightarrow 0$

$$
\tilde{\psi}_{q}^{h} \rightarrow \psi_{q} \quad W^{1, \infty} \text { weak- } .
$$


We also set

$$
\tilde{w}_{q}^{h}(t)=u_{J_{q}^{h}(n)+\epsilon_{q}^{h}(n)}^{n} \quad \text { for } \quad t_{n} \leq t<t_{n+1} .
$$

Using (3.17) and (3.7) of $w_{q}^{h}$, we can check that

$$
\tilde{w}_{q}^{h} \rightarrow w_{q} \quad L^{1} \text { strongly. }
$$

Consider

$$
I^{h}(\theta) \equiv-\sum_{(n, j) \in \mathcal{P}_{q}^{h}}\left(u_{j}^{n+1}-u_{j}^{n}+\lambda\left(g_{j+1 / 2}^{n}-g_{j-1 / 2}^{n}\right)\right) \theta_{j}^{n} h=0,
$$

which vanishes identically in view of (2.1). Using summation by parts gives

$$
\begin{aligned}
I^{h}(\theta)= & -\sum_{(n, j) \in \mathcal{P}_{q}^{h}}\left(u_{j}^{n+1} \theta_{j}^{n+1}-u_{j}^{n} \theta_{j}^{n}\right) h+\sum_{n} g_{J_{q}^{h}(n)+\epsilon_{q}^{h}(n)-1 / 2}^{n} \theta_{J_{q}^{h}(n)}^{n} \tau \\
& +\sum_{(n, j) \in \mathcal{P}_{q}^{h}} u_{j}^{n+1}\left(\theta_{j}^{n+1}-\theta_{j}^{n}\right) h+g_{j+1 / 2}^{n}\left(\theta_{j+1}^{n}-\theta_{j}^{n}\right) \tau \\
= & I_{1}^{h}(\theta)+I_{2}^{h}(\theta)+I_{3}^{h}(\theta) .
\end{aligned}
$$

The passage to the limit in $I_{3}^{h}(\theta)$ is an easy matter, since it has the classical form met, for instance, in the Lax-Wendroff theorem. We find that

$$
I_{3}^{h}(\theta) \rightarrow I_{3}(\theta)=\iint_{\left\{x \geq \psi_{q}(t)\right\}}\left(v \partial_{t} \theta+f(v) \partial_{x} \theta\right) d x d t .
$$

To deal with $I_{2}^{h}(\theta)$, we recall that the flux $g_{J_{q}^{h}(n)+\epsilon_{q}^{h}(n)-1 / 2}^{n}$ depends on two arguments, so satisfies

$$
\begin{aligned}
g_{J_{q}^{h}(n)+\epsilon_{q}^{h}(n)-1 / 2}^{n} & =f\left(u_{J_{q}^{h}(n)+\epsilon_{q}^{h}(n)}^{n}\right)+O\left(\left|u_{J_{q}^{h}(n)-1+\epsilon_{q}^{h}(n)}^{n}-u_{J_{q}^{h}(n)+\epsilon_{q}^{h}(n)}^{n}\right|\right) \\
& =f\left(\tilde{w}_{q}^{h}\left(t_{n}\right)\right)+O\left(\left|u_{J_{q}^{h}(n)-1+\epsilon_{q}^{h}(n)}^{n}-u_{J_{q}^{h}(n)+\epsilon_{q}^{h}(n)}^{n}\right|\right) .
\end{aligned}
$$

Indeed, by construction, the point $J_{q}^{h}(n)+\epsilon_{q}^{h}(n)-1 / 2$ is an end point of a cell achieving an extremum value: One has $\epsilon_{q}^{h}(n) \in\{0,1\}$, so $J_{q}^{h}(n)+\epsilon_{q}^{h}(n)-1 / 2 \in$ $\left\{J_{q}^{h}(n)-1 / 2, J_{q}^{h}(n)+1 / 2\right\}$. Using (3.26), estimate (3.17), the Cauchy-Schwarz inequality, and the Lebesgue convergence theorem, it is not hard to prove that

$$
I_{2}^{h}(\theta) \rightarrow I_{2}(\theta)=\int_{\mathbb{R}_{+}} f\left(w_{q}(t)\right) \theta\left(\psi_{q}(t), t\right) d t .
$$

It remains to prove that

$$
I_{1}^{h}(\theta) \rightarrow I_{1}(\theta)=-\int_{\mathbb{R}_{+}} \frac{d \psi_{q}}{d t}(t) w_{q}(t) \theta\left(\psi_{q}(t), t\right) d t .
$$

We return to the definition of the modified path and define $e_{q}^{h}(n+1)$ by

$$
J_{q}^{h}(n+1)+\epsilon_{q}^{h}(n+1)=J_{q}^{h}(n)+\epsilon_{q}^{h}(n)+e_{q}^{h}(n+1) .
$$

Using only (3.21), one can prove that

$$
\sum_{n} e_{q}^{h}(n) u_{J_{q}^{h}(n)+\epsilon_{q}^{h}(n)}^{n} \theta_{J_{q}^{h}(n)+\epsilon_{q}^{h}(n)}^{n} h \rightarrow-\int_{\mathbb{R}_{+}} \frac{d \psi_{q}}{d t}(t) \theta\left(\psi_{q}(t), t\right) d t .
$$

We claim that

$$
e_{q}^{h}(n+1) \in\{-1,0,1\}
$$


Namely, using (2.11) and the definition of $\epsilon_{q}^{h}(n+1)$, we have either

$$
\epsilon_{q}^{h}(n)=1, \quad J_{q}^{h}(n+1)=J_{q}^{h}(n) \text { or } J_{q}^{h}(n+1)+1, \text { and } e_{q}^{h}(n+1)=1 \text { or } 1
$$

or

$$
\epsilon_{q}^{h}(n)=1, \quad J_{q}^{h}(n+1)=J_{q}^{h}(n) \text { or } J_{q}^{h}(n+1)+1 \text {, and } e_{q}^{h}(n+1)=-1 \text { or } 0 .
$$

The term $I_{1}^{h}(\theta)$ then can be rewritten in the form

$$
\begin{aligned}
I_{1}^{h}(\theta) & =\sum_{j \geq J_{q}^{h}(n)+\epsilon_{q}^{h}(n)} u_{j}^{n+1} \theta_{j}^{n+1} h-\sum_{j \geq J_{q}^{h}(n)+\epsilon_{q}^{h}(n)} u_{j}^{n} \theta_{j}^{n} h \\
& =\sum_{j \geq J_{q}^{h}(n-1)+\epsilon_{q}^{h}(n-1)} u_{j}^{n} \theta_{j}^{n} h-\sum_{j \geq J_{q}^{h}(n)+\epsilon_{q}^{h}(n)} u_{j}^{n} \theta_{j}^{n} h,
\end{aligned}
$$

so that

$$
\begin{aligned}
I_{1}^{h}(\theta)=- & \sum_{e_{q}^{h}(n)=-1} u_{J_{q}^{h}(n)+\epsilon_{q}^{h}(n)}^{n} \theta_{J_{q}^{h}(n)+\epsilon_{q}^{h}(n)}^{n} h \\
& +\sum_{e_{q}^{h}(n)=1} u_{J_{q}^{h}(n)+\epsilon_{q}^{h}(n)-1}^{n} \theta_{J_{q}^{h}(n)+\epsilon_{q}^{h}(n)-1}^{n} h .
\end{aligned}
$$

Observe that, in the second sum above, $J_{q}^{h}(n)+\epsilon_{q}^{h}(n)-1=J_{q}^{h}(n-1)+\epsilon_{q}^{h}(n-1)$, and consider the decomposition $I_{1}^{h}(\theta)=I_{1,1}^{h}(\theta)+I_{1,2}^{h}(\theta)$ with

$$
I_{1,1}^{h}(\theta)=\sum_{e_{q}^{h}(n)=1}\left(u_{J_{q}^{h}(n)+\epsilon_{q}^{h}(n)-1}^{n} \theta_{J_{q}^{h}(n)+\epsilon_{q}^{h}(n)-1}^{n}-u_{J_{q}^{h}(n)+\epsilon_{q}^{h}(n)}^{n} \theta_{J_{q}^{h}(n)+\epsilon_{q}^{h}(n)}^{n}\right) h
$$

and

$$
I_{1,2}^{h}(\theta)=\sum_{e_{q}^{h}(n)=1} u_{J_{q}^{h}(n)+\epsilon_{q}^{h}(n)}^{n} \theta_{J_{q}^{h}(n)+\epsilon_{q}^{h}(n)}^{n} h-\sum_{e_{q}^{h}(n)=-1} u_{J_{q}^{h}(n)+\epsilon_{q}^{h}(n)}^{n} \theta_{J_{q}^{h}(n)+\epsilon_{q}^{h}(n)}^{n} h .
$$

On one hand, we have

$$
\begin{aligned}
I_{1,1}^{h}(\theta) \leq & \sum_{e_{q}^{h}(n)=1}\left|u_{J_{q}^{h}(n)+\epsilon_{q}^{h}(n)-1}^{n} \theta_{J_{q}^{h}(n)+\epsilon_{q}^{h}(n)-1}^{n}-u_{J_{q}^{h}(n)+\epsilon_{q}^{h}(n)}^{n} \theta_{J_{q}^{h}(n)+\epsilon_{q}^{h}(n)}^{n}\right| h \\
\leq & O(1) \sum_{e_{q}^{h}(n)=1}\left|u_{J_{q}^{h}(n)+\epsilon_{q}^{h}(n)-1}^{n}-u_{J_{q}^{h}(n)+\epsilon_{q}^{h}(n)}^{n}\right| h \\
& \quad+O(1) \sum_{e_{q}^{h}(n)=1}\left|\theta_{J_{q}^{h}(n)+\epsilon_{q}^{h}(n)-1}^{n}-\theta_{J_{q}^{h}(n)+\epsilon_{q}^{h}(n)}^{n}\right| h,
\end{aligned}
$$

and, in view of (3.17) and the smoothness property of $\theta$,

$$
\begin{aligned}
I_{1,1}^{h}(\theta) & \leq O(1) h^{1 / 2}\left(\sum_{n}\left|u_{J_{q}^{h}(n)-1}^{n}-u_{J_{q}^{h}(n)}^{n}\right|^{2}\right)^{1 / 2}+O(1) h \\
& \leq O(1)\left(h+h^{1 / 2}\right) \leq O(1) h^{1 / 2}
\end{aligned}
$$

which implies

$$
I_{1,1}^{h}(\theta) \rightarrow 0 \quad \text { as } h \rightarrow 0 .
$$


The expression for $I_{1,2}^{h}(\theta)$ can be simplified, namely

$$
I_{1,2}^{h}(\theta)=-\sum_{n} e_{q}^{h}(n) u_{J_{q}^{h}(n)+\epsilon_{q}^{h}(n)}^{n} \theta_{J_{q}^{h}(n)+\epsilon_{q}^{h}(n)}^{n} h .
$$

Using (3.29), we find that

$$
I_{1,2}^{h}(\theta) \rightarrow I_{1}(\theta) \quad \text { as } h \rightarrow 0
$$

In view of (3.25), (3.27), and (3.28), we conclude that

$$
I_{1}(\theta)+I_{2}(\theta)+I_{3}(\theta)=0 .
$$

Finally, using in (3.33) a sequence of test-functions $\theta$, whose supports shrink and concentrate on the curve $\psi_{q}$, the desired Rankine-Hugoniot relation (3.20) with the "+" sign follows at the limit. This completes the proof of Lemma 3.8.

Proof of Lemma 3.9. For definiteness, we assume that $u_{J_{q}^{h}(n)}^{n}$ is a maximum value and that

$$
\min \left(u_{J_{q}^{h}(n)}^{n}-u_{J_{q}^{h}(n) \pm 1}^{n}\right)=u_{J_{q}^{h}(n)}^{n}-u_{J_{q}^{h}(n)-1}^{n} .
$$

The other cases are treated similarly. To simplify the notation, set $j_{*}=J_{q}^{h}(n)$. By the uniform decay property $(2.12)$, we have

$$
u_{j_{*}}^{n}-u_{j_{*}}^{n+1} \geq \alpha\left(u_{j_{*}}^{n}-u_{j_{*}-1}^{n}\right)^{2} .
$$

By the strong maximum principle (2.11), we have

$$
u_{j_{*}-1}^{n+1}-u_{j_{*}-1}^{n} \leq \frac{1}{2}\left(u_{j_{*}}^{n}-u_{j_{*}-1}^{n}\right) .
$$

From (3.35), we deduce that

$$
\begin{aligned}
u_{j_{*}-1}^{n+1} & \leq u_{j_{*}-1}^{n}+\frac{1}{2}\left(u_{j_{*}}^{n}-u_{j_{*}-1}^{n}\right) \\
& =u_{j_{*}}^{n}-\frac{1}{2}\left(u_{j_{*}}^{n}-u_{j_{*}-1}^{n}\right) \\
& \leq u_{j_{*}}^{n}-\frac{1}{2} \min \left(\left(u_{j_{*}}^{n}-u_{j_{*}-1}^{n}\right),\left(u_{j_{*}}^{n}-u_{j_{*}-1}^{n}\right)^{2}\right)
\end{aligned}
$$

Note that $u_{j_{*}}^{n}-u_{j_{*}-1}^{n}$ might be either $\leq 1$ or $\geq 1$. Then we get

$$
u_{j_{*}-1}^{n+1} \leq u_{j_{*}}^{n}-\beta \min \left(\left(u_{j_{*}}^{n}-u_{j_{*}-1}^{n}\right),\left(u_{j_{*}}^{n}-u_{j_{*}-1}^{n}\right)^{2}\right) .
$$

On the other hand, (3.34) can be written in the form

$$
u_{j_{*}}^{n+1} \leq u_{j_{*}}^{n}-\alpha\left(u_{j *}^{n}-u_{j_{*}-1}^{n}\right)^{2} .
$$

Moreover, using (2.11) again, we obtain

$$
u_{j_{*}+1}^{n+1} \leq u_{j_{*}+1}^{n}+\frac{1}{2}\left(u_{j_{*}}^{n}-u_{j_{*}+1}^{n}\right) \leq u_{j_{*}}^{n}+\frac{1}{2}\left(u_{j_{*}}^{n}-u_{j_{*}-1}^{n}\right) .
$$

Hence, in view of (3.36),

$$
u_{j_{*}+1}^{n+1} \leq u_{j_{*}}^{n}-\beta \min \left(\left(u_{j_{*}}^{n}-u_{j_{*}-1}^{n}\right),\left(u_{j_{*}}^{n}-u_{j_{*}-1}^{n}\right)^{2}\right) .
$$

It follows from (3.37)-(3.39) that

$$
\max \left(u_{j_{*}-1}^{n+1}, u_{j_{*}}^{n+1}, u_{j_{*}+1}^{n+1}\right) \leq u_{j_{*}}^{n}-\beta \min \left(\left(u_{j_{*}}^{n}-u_{j_{*}-1}^{n}\right),\left(u_{j_{*}}^{n}-u_{j_{*}-1}^{n}\right)^{2}\right),
$$

and thus

$$
u_{J_{q}^{h}(n+1)}^{n+1} \leq u_{j_{*}}^{n}-\beta \min \left(\left(u_{j_{*}}^{n}-u_{j_{*}-1}^{n}\right),\left(u_{j_{*}}^{n}-u_{j_{*}-1}^{n}\right)^{2}\right),
$$


since $u_{J_{q}^{h}(n+1)}^{n+1}$ by definition achieves the maximum in (3.20). Finally, we have proved that

$$
u_{J_{q}^{h}(n)}^{n}-u_{J_{q}^{h}(n+1)}^{n+1} \geq \beta \min \left(\left(u_{j_{*}}^{n}-u_{j_{*}-1}^{n}\right),\left(u_{j_{*}}^{n}-u_{j_{*}-1}^{n}\right)^{2}\right) .
$$

By assumption, $u_{j_{*}}^{n}-u_{j_{*}-1}^{n} \leq u_{j_{*}}^{n}-u_{j_{*}}^{n}$. Thus (3.42) gives (3.17) after summation w.r.t. $n$.

Lemma 3.10. We have

$$
v(x-, t) \geq v(x+, t) \quad \text { in the set } \Omega_{3}(v) .
$$

The proof is based on the fact that one of the entropy criteria is satisfied on each side of a path: the Lax shock inequality on the non-increasing side, and the cell entropy inequality on the non-decreasing one.

Proof. We claim that, along any path of extremum $\psi_{q}$,

$$
v\left(\psi_{q}(t)-, t\right) \geq v\left(\psi_{q}(t)+, t\right) \quad \text { for a.e. } t \in\left(0, T_{q}\right) .
$$

We use the notation introduced in the proof of Lemma 3.8. A new difficulty arises: several paths may accumulate in a region. Lemma 3.8 was concerned with the discrete conservation law (2.1), which holds in both the non-increasing and nondecreasing regions. For the entropy consistency, we do not use the same criterion, and this complicates the proof.

To begin with, consider a path $\psi_{q}$ and a point $\left(\psi_{q}\left(t_{0}\right), t_{0}\right)$, that is supposed to be an "isolated" point of change of monotonicity, in the sense that $\psi_{q-1}\left(t_{0}\right)<$ $\psi_{q}\left(t_{0}\right)<\psi_{q+1}\left(t_{0}\right)$. By continuity, these inequalities then hold with $t_{0}$ replaced by any $t$ lying in a small neighborhood of $t_{0}$. For definiteness, we also suppose that $\psi_{q}$ is a path of minimum values. We later analyze the case that two or more paths of extrema accumulate in a neighborhood of $\left(\psi_{q}\left(t_{0}\right), t_{0}\right)$.

Let $\theta$ be a non-negative test function of the two variables $(x, t)$ having its support included in a small neighborhood of $\left(\psi_{q}\left(t_{0}\right), t_{0}\right)$. We can always assume that $u^{h}$ is non-increasing on the left side of the curve $\psi_{q}^{h}$, and non-decreasing on the right side. Using the notation introduced in the proof of Lemma 3.8, we aim at passing to the limit in

$$
I^{h}(\theta)=-\sum_{(j, n) \in \mathcal{P}_{q}^{h}}\left(U_{j}^{n+1}-U_{j}^{n}+\lambda\left(G_{j+1 / 2}^{n}-G_{j-1 / 2}^{n}\right)\right) \theta_{j}^{n} h \geq 0 .
$$

Note that $I^{h}(\theta)$ is non-positive, according to the cell entropy inequality (2.9) and since the $u^{h}$ 's are non-decreasing on the right side.

Integrating by parts in $I^{h}(\theta)$ gives

$$
\begin{aligned}
I^{h}(\theta)=- & \sum_{(j, n) \in \mathcal{P}_{q}^{h}}\left(U_{j}^{n+1} \theta_{j}^{n+1}-U_{j}^{n} \theta_{j}^{n}\right) h \\
& +\sum_{n} G_{J_{q}^{h}(n)+\epsilon_{q}^{h}(n)-1 / 2}^{n} \theta_{J_{q}^{h}(n)+\epsilon_{q}^{h}(n)-1 / 2} \tau \\
& +\sum_{(j, n) \in \mathcal{P}_{q}^{h}} U_{j}^{n+1}\left(\theta_{j}^{n+1}-\theta_{j}^{n}\right) h+G_{j+1 / 2}^{n}\left(\theta_{j+1}^{n}-\theta_{j}^{n}\right) \tau \\
= & I_{1}^{h}(\theta)+I_{2}^{h}(\theta)+I_{3}^{h}(\theta) .
\end{aligned}
$$


The passage to the limit in the term $I_{3}^{h}(\theta)$ is a classical matter. The treatment of $I_{2}^{h}(\theta)$ and $I_{1}^{h}(\theta)$ is similar to what was done to prove (3.27) and (3.28), respectively. Therefore we have

$$
\begin{gathered}
I_{1}^{h}(\theta) \rightarrow I_{1}(\theta)=-\int_{\mathbb{R}_{+}} \frac{d \psi_{q}}{d t}(t) U\left(w_{q}(t)\right) \theta\left(\psi_{q}(t), t\right) d t, \\
I_{2}^{h}(\theta) \rightarrow I_{2}(\theta)=\int_{\mathbb{R}_{+}} F\left(w_{q}(t)\right) \theta\left(\psi_{q}(t), t\right) d t, \\
I_{3}^{h}(\theta) \rightarrow I_{3}(\theta)=\iint_{\left\{x \geq \psi_{q}(t)\right\}}\left(U(v) \partial_{t} \theta+F(v) \partial_{x} \theta\right) d x d t .
\end{gathered}
$$

It follows from (3.46)-(3.48) that

$$
I_{1}(\theta)+I_{2}(\theta)+I_{3}(\theta) \geq 0 .
$$

Finally, using a sequence of test functions whose supports shrink and concentrate on the curve $\psi_{q}$, we deduce from (3.49) that the entropy dissipation is non-positive along the path, i.e.

$$
-\frac{d \psi_{q}}{d t}(t)\left(U\left(v\left(\psi_{q}(t)+, t\right)\right)-U\left(w_{q}(t)\right)\right)+F\left(v\left(\psi_{q}(t)+, t\right)\right)-F\left(w_{q}(t)\right) \leq 0 .
$$

Combined with (3.20), this inequality is equivalent to

$$
\left.v\left(\psi_{q}(t)+, t\right)\right) \leq w_{q}(t)
$$

which yields the desired inequality (3.44).

Consider next the case that several paths accumulate in the neighborhood of $\left(\psi_{q}\left(t_{0}\right), t_{0}\right)$. In view of (1.4), a finite number of paths only can accumulate at a given point. For definiteness we suppose that

- the point $\left(\psi_{q}\left(t_{0}\right), t_{0}\right)$ is a point of minimum values for $u^{h}$;

- the curves $\psi_{q+1}$ and $\psi_{q+2}$ coincide with $\psi_{q}$ in a neighborhood of $t_{0}$;

- and we have $\psi_{q-1}<\psi_{q}$ and $\psi_{q+2}<\psi_{q+3}$ in a neighborhood of $t_{0}$.

Suppose that, for instance, $w_{q}(t) \leq w_{q+2}(t)$. The other cases are treated similarly. In this situation, we are going to prove that

$$
v\left(\psi_{q}(t)-, t\right) \geq w_{q}(t)=w_{q+1}(t) \geq w_{q+2}(t)=v\left(\psi_{q}(t)+, t\right),
$$

at those points $t$ near $t_{0}$ where $\psi_{q}(t)=\psi_{q+1}(t)=\psi_{q+2}(t)$. Of course, (3.41) is a much stronger statement than (3.34). By definition of the paths of extrema, we have

$$
\begin{aligned}
& w_{q}(t) \leq w_{q+1}(t) \quad \text { and } \quad w_{q+1}(t) \geq w_{q+2}(t), \\
& v\left(\psi_{q}(t)-, t\right) \geq w_{q}(t) \quad \text { and } \quad w_{q+2}(t) \leq v\left(\psi_{q}(t)+, t\right) .
\end{aligned}
$$

Lemma 3.8 shows that

$$
w_{q}(t), w_{q+1}(t), w_{q+1}(t) \in\left\{v\left(\psi_{q}(t)-, t\right), v\left(\psi_{q}(t)+, t\right)\right\} .
$$

Thus, in order to get (3.50), it is sufficient to check the following two inequalities:

$$
\begin{gathered}
w_{q+2}(t) \geq v\left(\psi_{q}(t)+, t\right), \\
w_{q}(t) \geq w_{q+1}(t) .
\end{gathered}
$$

On one hand, the argument used in the first part of the present proof applies directly to the path $\psi_{q+2}(t)$ and the region located to the right of this curve, 
since $\psi_{q+2}(t)<\psi_{q+3}(t)$ in a neighborhood of $t_{0}$. As a consequence, we obtain $w_{q+2}(t) \geq v\left(\psi_{q+2}(t)+, t\right)$, which is exactly (3.51), since $\psi_{q+2}=\psi_{q}$.

On the other hand, to prove (3.52), let $\mathcal{P}_{q, q+1}^{h}$ be the (small) region limited by the curves $\psi_{q}^{h}$ and $\psi_{q+1}^{h}$ for $t$ belonging to a small neighborhood of $t_{0}$. Specifically, $\mathcal{P}_{q, q+1}^{h}$ is a set of indices of the form $(j, n)$ defined along the lines of the proof of Lemma 3.8. In particular, both paths are modified according to estimate (3.17), as explained before. Consider

$$
I^{h}(\theta)=-\sum_{(j, n) \in \mathcal{P}_{q, q+1}^{h}}\left(U_{j}^{n+1}-U_{j}^{n}+\lambda\left(G_{j+1 / 2}^{n}-G_{j-1 / 2}^{n}\right)\right) \theta_{j}^{n} h \geq 0 .
$$

Note that $I^{h}(\theta)$ is non-negative and

$$
\begin{aligned}
I^{h}(\theta)=- & \sum_{(j, n) \in \mathcal{P}_{q, q+1}^{h}}\left(U_{j}^{n+1} \theta_{j}^{n+1}-U_{j}^{n} \theta_{j}^{n}\right) h \\
& +\sum_{n} G_{J_{q}^{h}(n)+\epsilon_{q}^{h}(n)-1 / 2}^{n} \theta_{J_{q}^{h}(n)+\epsilon_{q}^{h}(n)-1 / 2}^{n} \tau \\
& -\sum_{n} G_{J_{q+1}^{h}(n)+\epsilon_{q+1}^{h}(n)-1 / 2}^{n} \theta_{J_{q+1}^{h}(n)+\epsilon_{q+1}^{h}(n)-1 / 2}^{n} \tau \\
& +\sum_{(j, n) \in \mathcal{P}_{q, q+1}^{h}} U_{j}^{n+1}\left(\theta_{j}^{n+1}-\theta_{j}^{n}\right) h+G_{j+1 / 2}^{n}\left(\theta_{j+1}^{n}-\theta_{j}^{n}\right) \tau \\
= & I_{1}^{h}(\theta)+I_{2, q}^{h}(\theta)-I_{2, q+1}^{h}(\theta)+I_{3}^{h}(\theta) .
\end{aligned}
$$

Using the technique developed for the proof of Lemma 3.8, we get

$$
\begin{aligned}
& I_{1}^{h}(\theta) \rightarrow I_{1}(\theta)=- \int_{\mathbb{R}_{+}} \frac{d \psi_{q}}{d t}(t) U\left(w_{q}(t)\right) \theta\left(\psi_{q}(t), t\right) d t \\
&+\int_{\mathbb{R}_{+}} \frac{d \psi_{q+1}}{d t}(t) U\left(w_{q+1}(t)\right) \theta\left(\psi_{q+1}(t), t\right) d t, \\
& I_{2, q}^{h}(\theta) \rightarrow I_{2, q}(\theta)= \int_{\mathbb{R}_{+}} F\left(w_{q}(t)\right) \theta\left(\psi_{q}(t), t\right) d t, \\
& I_{2, q+1}^{h}(\theta) \rightarrow I_{2, q+1}(\theta)=- \int_{\mathbb{R}_{+}} F\left(w_{q+1}(t)\right) \theta\left(\psi_{q+1}(t), t\right) d t, \\
& I_{3}^{h}(\theta) \rightarrow 0 .
\end{aligned}
$$

It follows that

$$
I_{1}(\theta)+I_{2, q}(\theta)+I_{2, q+1}(\theta) \geq 0
$$

which, since $\psi_{q}=\psi_{q+1}$ near $t_{0}$, is equivalent to the jump condition

$$
-\frac{d \psi_{q}}{d t}(t)\left(U\left(w_{q+1}(t)\right)-U\left(w_{q}(t)\right)\right)+F\left(w_{q+1}(t)\right)-F\left(w_{q}(t)\right) \leq 0
$$

which gives (3.52). This completes the proof of Lemma 3.10.

It is a classical matter to check that the initial condition (1.2) is satisfied by using (2.2) and the uniform BV bound. Since the function $v(t)$ has bounded total variation, it admits left and right traces at each point, and (1.5) and (1.6) are known to be equivalent at a point of discontinuity. Therefore the following result follows from Volpert's proof in [37]. 
Lemma 3.11. Supose $v$ is a function of bounded variation and a weak solution to the conservation law (1.1), and satisfies the initial condition (1.2) and the inequalities (3.14), (3.15), and (3.34), where $\Omega_{1}(v) \cup \Omega_{2}(v) \cup \Omega_{3}(v)=\mathbb{R} \times \mathbb{R}_{+}$. Then $v$ is the unique entropy solution to (1.1)-(1.2).

The proof of Theorem 2.3 is now complete.

The limiting paths $\psi_{q}$ associated with the scheme determine a decomposition of the plane into non-increasing/non-decreasing regions for the exact solution $u$. Such a decomposition is not unique, in general. Consider the decomposition found in the Appendix for the function $v$ and the corresponding paths $\varphi_{p}$. When $v$ is not constant in any neighborhood of an extremum path $\varphi_{p}$, the path is unique and must coincide with one of the paths $\psi_{q}$. When $v$ is constant in the neighborhood of a path $\varphi_{p}$, then the path may be arbitrarily modified and it may happen that no limiting path $\psi_{q}$ coincides with $\varphi_{p}$.

\section{Applichtion to the MUSCL scheme}

The purpose of this section is to apply Theorem 2.3 to van Leer's MUSCL scheme (for Monotone Upstream Scheme for Conservation Laws); cf. [23], [24]. This section also provides a proof of estimate (2.12) stated in Proposition 2.1, a new property of the Godunov scheme which does also hold for the MUSCL scheme.

It is convenient to formulate (2.1) in terms of the incremental coefficients $C_{j+1 / 2}^{ \pm, n}$ defined by

$$
C_{j+1 / 2}^{+, n}=-\lambda \frac{g_{j+1 / 2}^{n}-f\left(u_{j}^{n}\right)}{u_{j+1}^{n}-u_{j}^{n}}, \quad C_{j-1 / 2}^{-, n}=\lambda \frac{f\left(u_{j}^{n}\right)-g_{j-1 / 2}^{n}}{u_{j}^{n}-u_{j-1}^{n}},
$$

so that

$$
u_{j}^{n+1}=u_{j}^{n}+C_{j+1 / 2}^{+, n}\left(u_{j+1}^{n}-u_{j}^{n}\right)+C_{j-1 / 2}^{-, n}\left(u_{j-1}^{n}-u_{j}^{n}\right) .
$$

The numerical viscosity coefficient (cf. Tadmor [35]) being defined by

$$
Q_{j+1 / 2}^{n}=C_{j+1 / 2}^{+, n}+C_{j+1 / 2}^{-, n},
$$

the viscous form of the scheme is

$$
\begin{aligned}
u_{j}^{n+1}=u_{j}^{n} & -\frac{\lambda}{2}\left(f\left(u_{j+1}^{n}\right)-f\left(u_{j-1}^{n}\right)\right)+\frac{1}{2} Q_{j+1 / 2}^{n}\left(u_{j+1}^{n}-u_{j}^{n}\right) \\
& -\frac{1}{2} Q_{j-1 / 2}^{n}\left(u_{j-1}^{n}-u_{j}^{n}\right) .
\end{aligned}
$$

Proposition 4.1. The scheme (2.1) satisfies the local maximum principle (2.11) provided

$$
C_{j+1 / 2}^{+, n} \geq 0, \quad C_{j-1 / 2}^{-, n} \geq 0, \quad \text { and } \quad C_{j+1 / 2}^{+, n}+C_{j-1 / 2}^{-, n} \leq \frac{1}{2} .
$$

A sufficient condition for (4.4) to hold is

$$
C_{j+1 / 2}^{ \pm, n} \geq 0 \quad \text { and } \quad Q_{j+1 / 2}^{n} \leq 1 / 4 .
$$

Namely, if (4.5) holds, then $0 \leq C_{j+1 / 2}^{+, n} \leq 1 / 4$ and $0 \leq C_{j+1 / 2}^{-, n} \leq 1 / 4$, so that (4.4) is satisfied. In particular, the Godunov and Engquist-Osher schemes satisfy (4.4) under the CFL condition (2.4). When the numerical flux is independent of $\lambda$, the second inequality in (4.5) is always satisfied provided $\lambda$ is small enough. 
The Lax-Friedrichs type schemes have a constant numerical viscosity $Q_{j+1 / 2}^{n} \equiv$ $Q$. For the original Lax-Friedrichs scheme $Q=1$. Proposition 4.1 applies provided $Q \leq 1 / 4$. Observe that the monotonicity property does fail when $Q \in(2 / 3,1]$ : take for instance $f \equiv 0$ and $u_{j}^{n} \equiv 0$ for all $j \neq 0$ but $u_{0}^{n}>0$. This initial data has one maximum point, and at the next time step

$$
u_{j}^{n+1}=\frac{Q}{2} u_{j+1}^{n}+(1-Q) u_{j}^{n}+\frac{Q}{2} u_{j-1}^{n}
$$

admits two maximum points $j=-1$ and $j=1$. A related observation was made by Tadmor in [35]: for $Q \leq 1 / 2$, better properties can be obtained for the LaxFriedrichs scheme.

Proof of Proposition 4.1. Inequalities (2.11) can be written in terms of the incremental coefficients, namely

$$
\begin{aligned}
\frac{1}{2} \min \left(\delta_{j+1}^{n}, \delta_{j}^{n}, \delta_{j-1}^{n}\right) & \leq C_{j+1 / 2}^{+, n} \delta_{j+1}^{n}+\left(\frac{1}{2}-C_{j+1 / 2}^{+, n}-C_{j-1 / 2}^{-, n}\right) \delta_{j}^{n}+C_{j-1 / 2}^{-, n} \delta_{j-1}^{n} \\
& \leq \frac{1}{2} \max \left(\delta_{j+1}^{n}, \delta_{j}^{n}, \delta_{j-1}^{n}\right)
\end{aligned}
$$

with

$$
\delta_{j+1}^{n}=u_{j+1}^{n}-u_{j}^{n}, \quad \delta_{j}^{n}=0, \quad \delta_{j-1}^{n}=u_{j-1}^{n}-u_{j}^{n} .
$$

If (4.4) holds, then

$$
\left.2 C_{j+1 / 2}^{+, n} \delta_{j+1}^{n}+\left(1-2 C_{j+1 / 2}^{+, n}-2 C_{j-1 / 2}^{-, n}\right) \delta_{j}^{n}+2 C_{j-1 / 2}^{-, n} \delta_{j-1}^{n}\right)
$$

is a convex combination of the $\delta_{j}$ 's. So (4.6) and therefore (2.11) follow.

We now introduce van Leer's scheme, composed of a reconstruction step based on the min-mod limitor and a resolution step based on the Godunov solver. We use the notation introduced in Section 2. For simplicity in the presentation, we normalize the flux to satisfy $f(0)=f^{\prime}(0)=0$. From the approximation $\left\{u_{j}^{n}\right\}$ at the time $t=t_{n}$, we construct a piecewise affine function

$$
\tilde{u}_{j}^{n}(x)=u_{j}^{n}+s_{j}^{n}\left(x-x_{j}\right) / h \quad \text { for } x \in\left(x_{j-1 / 2}, x_{j+1 / 2}\right),
$$

where the slope $s_{j}^{n}$ is

$$
s_{j}^{n}=\operatorname{minmod}\left(u_{j}-u_{j-1},\left(u_{j+1}-u_{j-1}\right) / 2, u_{j+1}-u_{j}\right)
$$

with

$$
\operatorname{minmod}(a, b, c)=\left\{\begin{array}{l}
\min (a, b, c) \quad \text { if } a>0, b>0, \text { and } c>0 \\
\max (a, b, c) \quad \text { if } a<0, b<0, \text { and } c<0 . \\
0 \quad \text { in all other cases. }
\end{array}\right.
$$

We introduce the notation

$$
u_{j+1 / 2-}^{n}=u_{j}^{n}+s_{j}^{n} / 2 \quad \text { and } \quad u_{j+1 / 2+}^{n}=u_{j+1}^{n}-s_{j+1}^{n} / 2 .
$$

Then the solution is updated with (2.1), where the numerical flux is defined depending upon the values of the reconstruction at the interfaces. 
(1) If either $0 \leq u_{j}^{n} \leq u_{j+1}^{n}$ or $0 \leq u_{j+1}^{n} \geq u_{j}^{n}$, then the numerical flux is defined by using the characteristic line traced backward from the point $\left(x_{j+1 / 2}, t_{n+1 / 2}\right)$. Since the latter has a positive slope, we set

$$
g_{j+1 / 2}^{n}=f\left(u_{j+1 / 2-}^{n}\right)+f^{\prime}\left(u_{j+1 / 2-}^{n}\right)\left(v-u_{j+1 / 2-}^{n}\right)
$$

with $v$ solving

$$
u_{j+1 / 2-}^{n}=v+\frac{\lambda}{2} f^{\prime}(v) s_{j}^{n} .
$$

(2) If either $u_{j}^{n} \leq u_{j+1}^{n} \leq 0$, or $u_{j+1 /}^{n} \leq u_{j}^{n} \leq 0$, then the backward characteristic has a negative slope and we set

$$
g_{j+1 / 2}^{n}=f\left(u_{j+1 / 2+}^{n}\right)+f^{\prime}\left(u_{j+1 / 2+}^{n}\right)\left(v-u_{j+1 / 2+}^{n}\right)
$$

with $v$ solving

$$
u_{j+1 / 2+}^{n}=v+\frac{\lambda}{2} f^{\prime}(v) s_{j+1}^{n} .
$$

(3) In all other cases we set

$$
g_{j+1 / 2}^{n}=f(0)=0
$$

Equations (4.10b) and (4.11b) can be solved explicitly for the Burgers equation, since then $f^{\prime}(u)=u$ is linear. Observe that the scheme reduces to first order at sonic points and extrema.

The main result of this section is:

Theorem 4.2. For $\lambda$ small enough, the MUSCL method defined by (4.7)-(4.12) is a generalized monotone scheme in the sense of Definition 2.2. When (1.3)-(1.4) hold, the scheme converges in the strong $L^{1}$ topology to the unique entropy solution of (1.1)-(1.2).

It would be interesting to extend Theorem 4.2 to higher-order methods such as the Woodward-Collela P.P.M. scheme.

Proof of Theorem 4.2. We have to check that the scheme satisfies the three conditions in Definition 2.2. We always assume that $\lambda$ is, at least, less than or equal to $1 / 4$.

Step 1. Local maximum principle.

Estimate (2.11) is easily obtained by applying Proposition 4.1 and relying on the convexity of the flux function $f$. We omit the details.

Step 2. Cell entropy inequality.

Consider a region where the sequence $\left\{u_{j}^{n}\right\}$ is non-decreasing. We will use the entropy pair $(U, F)$ with $U(u)=u^{2} / 2$ and $F^{\prime}(u)=u f^{\prime}(u)$. Define the numerical entropy flux by

$$
\begin{aligned}
& G_{j+1 / 2}^{n}=F\left(u_{j+1 / 2-}^{n}\right)+U^{\prime}\left(u_{j+1 / 2-}^{n}\right)\left(f(v)-f\left(u_{j+1 / 2-}^{n}\right)\right), \\
& G_{j+1 / 2}^{n}=F\left(u_{j+1 / 2+}^{n}\right)+U^{\prime}\left(u_{j+1 / 2+}^{n}\right)\left(f(v)-f\left(u_{j+1 / 2+}^{n}\right)\right),
\end{aligned}
$$

and

$$
G_{j+1 / 2}^{n}=0
$$


in cases (1), (2), and (3), respectively. Inequality (2.9) is checked by direct calculation, for $\lambda$ small enough. Observe that case (3) is obvious since our scheme then reduces to a first order, entropy consistent scheme.

For definiteness we treat case (1), i.e. $f^{\prime}>0$ for the values of $u$ under consideration. We view the left hand side of $(2.9)$ as a function of $w=u_{j-1 / 2-}, u=u_{j}^{n}$, $v=u_{j+1 / 2-}$ and the value $\tilde{w}$ defined as

$$
w=\tilde{w}+\frac{\lambda}{2} f^{\prime}(\tilde{w}) t
$$

where $t$ stands for the slope in the cell $j-1$. We also introduce $\tilde{v}$, defined by

$$
v=\tilde{v}+\frac{\lambda}{2} f^{\prime}(\tilde{v}) s
$$

with $s=2(v-u)$. Since the approximate solution is non-increasing, we have $\tilde{w} \leq w \leq 2 u-v \leq u \leq v \leq \tilde{v}$. Set

$$
\begin{aligned}
& \Omega(\tilde{w}, w, u, v ; \lambda) \\
& =U(\bar{u})-U(u)+\lambda\left[F(v)+U^{\prime}(v)(f(\tilde{v})-f(v))-F(w)-U^{\prime}(w)(f(\tilde{w})-f(w))\right],
\end{aligned}
$$

and

$$
\bar{u}=u-\lambda\left[f(v)+f^{\prime}(v)(\tilde{v}-v)-f(w)-f^{\prime}(w)(\tilde{w}-w)\right] .
$$

Observe that

$$
\partial_{\tilde{w}} \Omega=U^{\prime}(\bar{u}) \lambda f^{\prime}(w)-\lambda U^{\prime}(w) f^{\prime}(\tilde{w})
$$

and

$$
\partial_{\tilde{w}}^{2} \Omega=U^{\prime \prime}(\bar{u}) \lambda^{2} f^{\prime}(w)-\lambda U^{\prime}(w) f^{\prime \prime}(\tilde{w}) \leq-C \lambda|w|
$$

for $w>0$ and $\lambda$ small enough. Therefore $\Omega$ is a concave function in $\tilde{w}$, and

$$
\Omega(\tilde{w}, w, u, v ; \lambda) \leq \Omega(w, w, u, v ; \lambda)-(w-\tilde{w}) \partial_{\tilde{w}} \Omega(w, w, u, v ; \lambda)-C \lambda|w||\tilde{w}-w|^{2} .
$$

But

$$
\begin{aligned}
\partial_{\tilde{w}} \Omega(w, w, u, v ; \lambda) & =\lambda f^{\prime}(w)\left(U^{\prime}(\bar{u})-U^{\prime}(w)\right) \\
& =U^{\prime \prime}(\xi) \lambda f^{\prime}(w)\left[u-w-\lambda\left(f(v)+f^{\prime}(v)(\tilde{v}-v)-f(w)\right)\right] \\
& \leq C \lambda|u-w|
\end{aligned}
$$

for some $\xi>0$ and $\lambda$ small enough. This proves that

$$
\begin{aligned}
\Omega(\tilde{w}, w, u, v ; \lambda) & \leq \Omega(w, w, u, v ; \lambda)-C \lambda\left(|\tilde{w}-w||u-w|+|w||\tilde{w}-w|^{2}\right) \\
& \leq \Omega(w, w, u, v ; \lambda),
\end{aligned}
$$

and we now simply use the notation $\Omega(w, u, v ; \lambda)$.

Taylor-expanding $\Omega$ with respect to $\lambda$ shows that the dominant term is the first order coefficient in $\lambda$ given by

$$
\hat{\Omega}_{1}(w, u, v) \equiv-U^{\prime}(u)(f(v)-f(w))+F(v)-F(w),
$$

in which $w \leq 2 u-v \leq u \leq v$. Since

$$
\partial_{w} \hat{\Omega}_{1}(w, u, v)=\left(U^{\prime}(u)-U^{\prime}(w)\right) f^{\prime}(w) \geq C|w||u-w|,
$$

we have

$$
\hat{\Omega}_{1}(w, u, v) \leq \hat{\Omega}_{1}(2 u-v, u, v)-C|w| \int_{w}^{2 u-v}(u-z) d z
$$


SO

$$
\hat{\Omega}_{1}(w, u, v) \leq \hat{\Omega}_{1}(2 u-v, u, v)-C^{\prime}|w||2 u-v-w||u-w| .
$$

It remains to study $\hat{\Omega}_{1}(2 u-v, u, v)=\tilde{\Omega}_{1}(u, v)$ with $u \leq v$. We find that

$$
\begin{aligned}
\partial_{v} \tilde{\Omega}_{1}(u, v) & =\left(U^{\prime}(v)-U^{\prime}(u)\right) f^{\prime}(v)+\left(U^{\prime}(2 u-v)-U^{\prime}(u)\right) f^{\prime}(2 u-v) \\
& =(v-u)\left(f^{\prime}(v)-f^{\prime}(2 u-v)\right) \leq-C|u-v|^{2} .
\end{aligned}
$$

It follows that $\tilde{\Omega}(u, v)$ is a non-increasing function of $v$ for all $v \geq u$, and since it vanishes for $v=u$,

$$
\tilde{\Omega}_{1}(u, v) \leq-C^{\prime}|u-v|^{3} .
$$

This proves that the first order term in $\lambda$ in the expansion of the function $\Omega$ is negative.

The same arguments are now applied to the function $\Omega(\lambda)$ directly. We have

$$
\begin{aligned}
\left.\partial_{w} \Omega_{(} w, u, v ; \lambda\right) & =\left(U^{\prime}(\bar{u})-U^{\prime}(w)\right) \lambda f^{\prime}(w) \\
& =\lambda f^{\prime}(w) U^{\prime \prime}(\xi)\left(u-w-\lambda\left(f(v)+f^{\prime}(v)(\tilde{v}-v)-f(w)\right)\right) \\
& =\lambda f^{\prime}(w) U^{\prime \prime}(\xi)(u-w-\lambda O(1)(u-w)) \\
& \geq \lambda C|w||u-w| .
\end{aligned}
$$

Therefore,

$$
\Omega(w, u, v ; \lambda) \leq \Omega(2 u-v, u, v ; \lambda)-\lambda C^{\prime}|w||2 u-v-w||u-w| .
$$

Denote $\hat{\Omega}(u, v ; \lambda)=\Omega(2 u-v, u, v ; \lambda) ;$ then

$$
\hat{\Omega}(u, v ; \lambda)=U(\bar{u})-U(u)+\lambda\left[F(v)+U^{\prime}(v)(f(\tilde{v})-f(v))-F(2 u-v)\right],
$$

where

$$
\bar{u}=u-\lambda\left[f(v)+f^{\prime}(v)(\tilde{v}-v)-f(2 u-v)\right] .
$$

We easily compute that

$$
\begin{aligned}
\frac{\partial_{v} \hat{\Omega}(u, v ; \lambda)}{\lambda}=f^{\prime}(v)\left(U^{\prime}(v)-U^{\prime}(\bar{u})\right) \\
+f^{\prime}(2 u-v)\left(U^{\prime}(2 u-v)-U^{\prime}(\bar{u})\right)+\lambda A(u, v ; \lambda)
\end{aligned}
$$

with

$$
|A(u, v ; \lambda)| \leq C|u-v|^{2} .
$$

This establishes the desired conclusion for $\lambda$ small enough.

Step 3. Quadratic decay property.

Near a local extremum, the MUSCL scheme essentially reduces to the Godunov scheme. So it is enough to check the quadratic decay property (2.12) for the Godunov scheme. This can be done from the explicit formula (2.6).

The simplest situation is obtained with the Godunov scheme and when $f^{\prime}$ has a sign, say is positive. Assume $u_{j}^{n}$ is a local maximum. We have

$$
u_{j}^{n+1}=u_{j}^{n}-\lambda\left(f\left(u_{j}^{n}\right)-f\left(u_{j-1}^{n}\right)\right) \text {; }
$$


thus

$$
\begin{aligned}
u_{j}^{n}-u_{j}^{n+1} & =\lambda\left(f\left(u_{j}^{n}\right)-f\left(u_{j-1}^{n}\right)\right) \\
& \geq \lambda f^{\prime}\left(u_{j-1}^{n}\right)\left(u_{j}^{n}-u_{j-1}^{n}\right)+\lambda\left(\inf f^{\prime \prime} / 2\right)\left(u_{j}^{n}-u_{j-1}^{n}\right)^{2} \\
& \geq \lambda\left(\inf f^{\prime \prime} / 2\right) \min _{ \pm}\left(u_{j}^{n}-u_{j \pm 1}^{n}\right)^{2} .
\end{aligned}
$$

This establishes (2.11) when $f^{\prime}>0$.

It remains to treat the sonic case where $f^{\prime}$ has no definite sign. We will rely on the following technical remark. Given two points such that

$$
u_{-}<0<u_{+}, \quad f\left(u_{-}\right)=f\left(u_{+}\right),
$$

there exist $c_{1}, c_{2}>0$ (independent of $u_{ \pm}$) such that

$$
c_{1}\left|u_{-}\right| \leq\left|u_{+}\right| \leq c_{2}\left|u_{-}\right| .
$$

Considering the case $u_{j-1}^{n}<0<u_{j}^{n}$, and using Osher's formula for the Riemann problem, we have

$$
\begin{aligned}
u_{j}^{n}-u_{j}^{n+1} & =\lambda\left(\max _{\left(u_{j+1}^{n}, u_{j}^{n}\right)} f-\min _{\left(u_{j-1}^{n}, u_{j}^{n}\right)} f\right) \\
& =\lambda\left(\max _{\left(u_{j+1}^{n}, u_{j}^{n}\right)} f-f(0)\right) \\
& =\lambda\left(\inf f^{\prime \prime} / 2\right)\left(\max _{\left|u_{j+1}^{n}\right|,\left|u_{j}^{n}\right|} f\right)^{2} \\
& \geq c\left|u_{j+1}^{n}-u_{j}^{n}\right|^{2} .
\end{aligned}
$$

Consider next the case $0<u_{j-1}^{n}<u_{j}^{n}$; then

$$
\begin{aligned}
u_{j}^{n}-u_{j}^{n+1} & =\lambda\left(\max _{\left(u_{j+1}^{n}, u_{j}^{n}\right)} f-f\left(u_{j}^{n}\right)\right) \\
& \geq \lambda\left(\inf f^{\prime \prime} / 2\right) \min \left(\left|u_{j+1}^{n}-u_{j}^{n}\right|^{2},\left|u_{j}^{n}-u_{j-1}^{n}\right|^{2}\right) .
\end{aligned}
$$

This completes the proof of Theorem 4.2.

\section{ACKNOWLEDGMEnTs}

Most of this work was done in 1992 while both authors were Courant instructors at the Courant Institute of Mathematical Sciences, New York University. The authors are very grateful to Peter D. Lax for helpful remarks on a first draft of this paper.

\section{Appendix: Monotonicity Property}

In this appendix we brieffly discuss the monotonicity property together with more basic properties of entropy solutions to conservation laws, which go back to Kružkov [19] and Volpert [37]. In the paper by Keyfitz, [18], somewhat simpler proofs are available for piecewise Lipschitz continuous solutions. We are interested in the local versions of the properties, i.e. formulated in domains limited by characteristic curves. To cope with discontinuous solutions, we use the concept of generalized characteristic curves introduced for ordinary differential equations by Filippov [9] and developed in the context of conservation laws by Dafermos; see e.g. [7] and the references therein. We recall that, through any point $\left(x_{0}, t_{0}\right)$, there exists a funnel of forward and backward generalized characteristic curves, which fill up a domain $\left\{\xi^{m}(t) \leq x \leq \xi^{M}(t)\right\}$. Here $\xi^{m}$ (respectively $\xi^{M}$ ) is called the minimal 
(resp. maximal) characteristic curve originating at $\left(x_{0}, t_{0}\right)$. It is known [9], [7] that a characteristic, say $\xi$, is Lipschitz continuous and for almost every time $t>0$ satisfies

$$
\frac{d \xi}{d t}(t)= \begin{cases}f^{\prime}(u(\xi(t), t)) & \text { if } u_{-}(t)=u_{+}(t)=u(\xi(t), t) \\ \frac{f\left(u_{+}\right)-f\left(u_{-}\right)}{u_{+}-u_{-}} & \text {if } u_{-} \neq u_{+}\end{cases}
$$

where $u_{ \pm}=u(\xi(t) \pm, t)$. For our purposes, $f$ is strictly convex and there is a unique forward characteristic issuing from $\left(x_{0}\right), t_{0}$; and there is no need to distinguish between the minimal characteristic and the maximal one, with the exception of those points where $t_{0}=0$ and $u_{0}$ has an increasing jump at $x_{0}$; cf. [7].

Solutions $u$ to (1.1) are Lipschitz continuous in time with values in $L^{1}$ and, for all times $t, u(t)$ has bounded total variation in $x$.

The following properties follow from [37], [19] and the technique of generalized characteristic in [9], [7].

Proposition A.1. Let $u$ be the entropy solution to (1.1)-(1.2). Given $x_{1}$ and $x_{2}$ with $x_{1}<x_{2}$, consider the maximal forward characteristic $\xi_{1}^{u}(t)$ issuing from $\left(0, x_{1}\right)$ and the minimal forward characteristic $\xi_{2}^{u}(t)$ from $\left(0, x_{2}\right)$. For all times $t \geq 0, u$ satisfies

(1) the local maximum principle for all $t \leq s$ and $y \in\left(\xi_{1}^{u}(s), \xi_{2}^{u}(s)\right)$ :

$$
\inf _{\xi_{1}^{u}(t)<x<\xi_{2}^{u}(t)}\{u(x, t)\} \leq u(s, y) \leq \sup _{\xi_{1}^{u}(t)<x<\xi_{2}^{u}(t)}\{u(x, t)\},
$$

(2) the local $L^{1}$ contraction property:

$$
\frac{d}{d t} \int_{\max \left(\xi_{1}^{u}(t), \xi_{1}^{v}(t)\right)}^{\min \left(\xi_{2}^{u}(t), \xi_{2}^{v}(t)\right)}|u(x, t)-v(x, t)| d x \leq 0,
$$

(3) the local order preserving property:

$$
\begin{aligned}
& \text { if } u_{0}(x) \leq v_{0}(x) \text { for all } x \in\left(\max \left(\xi_{1}^{u}(0), \xi_{1}^{v}(0)\right), \min \left(\xi_{2}^{u}(0), \xi_{2}^{v}(0)\right)\right), \\
& \text { then } u(x, t) \leq v(x, t) \text { for all } x \in\left(\max \left(\xi_{1}^{u}(t), \xi_{1}^{v}(t)\right)<x<\min \left(\xi_{2}^{u}(t), \xi_{2}^{v}(t)\right)\right) \text {, }
\end{aligned}
$$

(4) and the local TVD property:

$$
\frac{d}{d t} \mathrm{TV}_{\xi_{1}^{u}(t)}^{\xi_{u}^{u}(t)} u(t) \leq 0
$$

where in (1) and (3) the function $v$ denotes the solution to (1.1)-(1.2) with $u_{0}$ replaced by a function $v_{0} \in B V(\mathbb{R})$, and the curves $\xi_{1}^{v}(t)$ and $\xi_{2}^{v}(t)$ are defined in the obvious way.

In (A.5), $\mathrm{TV}_{a}^{b}(w)$ denotes the total variation of a function $w:(a, b) \rightarrow \mathbb{R}$. The derivatives in (A.3) and (A.5) are to be understood in the distributional sense. At least with $x_{1}=\xi_{1}^{u}=-\infty$ and $x_{2}=\xi_{2}^{u}=\infty$, (A.5) is a direct consequence of (A.3) and the invariance by translation of the solution-operator for (1.1).

The following proposition concerns the monotonicity property, which is a refinement to the statement that the solution-operator is monotonicity preserving, i.e. satisfies

if $u_{0}$ is monotone, then $u(t)$ is monotone for all $t \geq 0$.

Namely, (A.6) follows from (A.3) by taking $x_{1}=\xi_{1}^{u}=-\infty$ and $x_{2}=\xi_{2}^{u}=\infty$ and $v_{0}(x)=u_{0}(x+y)$ for positive or negative values of $y$.

It is convenient now to assume that (1.4) is satisfied. 
Proposition A.2 (Monotonicity property). Suppose that the initial condition $u_{0}$ has a locally finite number of local extrema. There exist (Lipschitz continuous) generalized characteristic curves $\varphi_{q}:[0, \infty) \rightarrow \mathbb{R}$-the index $q$ describing a subset $E\left(u_{0}\right)$ of consecutive integers - such that

$$
\varphi_{q} \leq \varphi_{q+1}
$$

there are only a finite number of such curves in each compact set,

and, for all $t \geq 0$ and all relevant values of $p$,

$$
\begin{aligned}
& u(t) \text { is non-decreasing for } x \in\left(\varphi_{2 p}(t), \varphi_{2 p+1}(t)\right), \\
& u(t) \text { is non-increasing for } x \in\left(\varphi_{2 p-1}(t), \varphi_{2 p}(t)\right),
\end{aligned}
$$

and, as long as $\varphi_{2 p}(t) \neq \varphi_{2 p+1}(t)$,

$$
\begin{aligned}
& u\left(\varphi_{2 p}(t)+, t\right) \text { is non-decreasing, } \\
& u\left(\varphi_{2 p+1}(t)-, t\right) \text { is non-increasing. }
\end{aligned}
$$

The paths $\varphi_{2 p}$ and $\varphi_{2 p+1}$ are called a path of local maximum and a path of local minimum for $u$, respectively. By convention, $\varphi_{q} \equiv-\infty$ for $q>\max E\left(u_{0}\right)$ and $\varphi_{q} \equiv+\infty$ for $q<\min E\left(u_{0}\right)$.

If two initially distinct paths cross at a later time, then from that time they will coincide thanks to the uniqueness property for forward characteristics. Note also that such paths need no longer be paths of local extremum in a strict sense, but arbitrary characteristics, even though (A.9) would still hold. On the other hand, when simultaneously $u_{0}$ has a decreasing jump at a point $x_{0}$, that $u_{0}\left(x_{0}-\right)$ is a local maximum, and $u\left(x_{0}+\right)$ is a local minimum, then two equal paths originate from $\left(0, x_{0}\right)$, one being a path of minimum and the other a path of maximum. When simultaneously $u_{0}$ has an increasing jump at $x_{0}$, that $u_{0}\left(x_{0}-\right)$ is a local minimum, and that $u_{0}\left(x_{0}+\right)$ a local maximum, then two distinct paths originate from $\left(0, x_{0}\right)$.

Proposition A.2 is a classical matter in the literature, although no specific reference seems available. Cf. however Harten [13] and Tadmor [35], where the ideas are developed.

Proof of Proposition A.2. First of all, the points $\varphi_{q}(0)$ and the set $E\left(u_{0}\right)$ are defined from the initial condition $u_{0}$ in an obvious way so that the conditions (A.7)-(A.10) hold true at time $t=0$. Let us define $\varphi_{2 p}(t)$ to be the maximal forward characteristic issuing from $\varphi_{2 p}(0)$. Similarly, let $\varphi_{2 p+1}(t)$ be the minimal forward characteristics issuing from $\varphi_{2 p+1}(0)$. Indeed, one needs to distinguish between minimal and maximal characteristics only in the case of an initially increasing jump. It may happen that both a path of minimum and a path of maximum may originate from such a point of increasing jump.

Property (A.7) is an immediate consequence of the uniqueness property of the forward characteristic. Condition (A.8) follows from the property of propagation with finite speed satisfied by solutions to (1.1) and the fact that the initial data has a locally finite number of local extrema. Indeed, (A.1) yields a uniform bound for the slopes of the characteristics.

In order to establish (A.9) and (A.10), we first suppose that $u_{0}$ does not admit increasing jumps. Consider an interval of the form $\left(\varphi_{2 p}(t), \varphi_{2 p+1}(t)\right)$ for those values of $t$ when this interval is not empty. Note first that, taking $\xi_{1}^{u}=\varphi_{2 p}$ and 
$\xi_{2}^{u}=\varphi_{2 p+1}$, the local maximum principle (A.2) implies in particular that

$$
\begin{array}{r}
u\left(\varphi_{2 p}(t)+, t\right) \geq u_{0}\left(\varphi_{2 p}(0)+\right), \\
u\left(\varphi_{2 p+1}(t)-, t\right) \leq u_{0}\left(\varphi_{2 p+1}(0)-\right) .
\end{array}
$$

Let $w$ be the entropy solution to (1.1) associated with the initial condition

$$
w(x, 0)=w_{0}(x) \equiv \begin{cases}u_{0}\left(\varphi_{2 p}(0)+\right) & \text { if } x<\varphi_{2 p}(0), \\ u_{0}(x) & \text { if } \varphi_{2 p}(0)<x<\varphi_{2 p+1}(0), \\ u_{0}\left(\varphi_{2 p+1}(0)-\right) & \text { if } x>\varphi_{2 p+1}(0) .\end{cases}
$$

The data $w_{0}$ is non-decreasing and, in view of (A.6),

$$
\text { the solution } w \text { is non-decreasing for all times. }
$$

Let $\psi_{2 p}$ and $\psi_{2 p+1}$ be the forward characteristics associated with $w$ and issued $\varphi_{2 p}(0)$ and $\varphi_{2 p+1}(0)$ at time $t=0$, respectively. Observe that the maximum forward and the minimum forward curves coincide, since by construction $w_{0}$ is continuous at $\varphi_{2 p}(0)$ and $\varphi_{2 p+1}(0)$. Note in passing that the function $w$ satisfies

$$
w(x, t)= \begin{cases}u_{0}\left(\varphi_{2 p}(0)+\right) & \text { if } x<\psi_{2 p}(t), \\ u_{0}\left(\varphi_{2 p+1}(0)-\right) & \text { if } x>\psi_{2 p+1}(t) .\end{cases}
$$

Using (A.11) and (A.14) and the fact that $f^{\prime}($.$) is increasing, one gets$

$$
\frac{d \varphi_{2 p+1}}{d t}(t) \leq f^{\prime}\left(u\left(\varphi_{2 p+1}(t)-, t\right)\right) \leq f^{\prime}\left(u_{0}\left(\varphi_{2 p+1}(0)-\right)\right) \leq \frac{d \psi_{2 p+1}}{d t}(t),
$$

which implies

$$
\varphi_{2 p+1}(t) \leq \psi_{2 p+1}(t) .
$$

Similarly,

$$
\psi_{2 p}(t) \leq \varphi_{2 p}(t)
$$

Using the $L^{1}$ contraction principle (A.3), it follows that

$$
u=w \quad \text { for } \varphi_{2 p}(t)<x<\varphi_{2 p+1}(t),
$$

and, in view of (A.13), the function $u$ is non-decreasing and (A.9) holds. Using (A.9) and the local maximum principle (A.2) finally provides (A.10). The proof is complete in the case of an interval of the form $\left(\varphi_{2 p}(t), \varphi_{2 p+1}(t)\right)$. An interval $\left(\varphi_{2 p-1}(t), \varphi_{2 p}(t)\right)$ can be treated in a similar fashion.

It remains to consider increasing jumps in $u_{0}$. That situation can be treated by using the following property. Suppose $u_{0}$ has an increasing jump at a point $x_{0}$, and let $\varphi^{m}(t)$ and $\varphi^{M}(t)$ be the minimal and maximal forward curves from $x_{0}$. It is known that at least for small times the function $u(t)$ coincides with the rarefaction wave connecting the values $u_{0}\left(x_{0} \pm\right)$ in the interval $\left(\varphi^{m}(t), \varphi^{M}(t)\right)$.

This completes the proof of Proposition A.2.

\section{REFERENCES}

1. F. Bouchut, C. Bourdarias, and B. Perthame, A MUSCL methods satisfying all the numerical entropy inequalities, Math. Comp. 65 (1996), 1439-1461. MR 97a:65080

2. Y. Brenier and S.J. Osher, The one-sided Lipschitz condition for convex scalar conservation laws, SIAM J. Numer. Anal. 25 (1988), 8-23. MR 89a:65134

3. E. Conway and J.A. Smoller, Global solutions of the Cauchy problem for quasilinear first order equations in several space variables, Comm. Pure Appl. Math. 19 (1966), 95-105. MR 33:388 
4. F. Coquel and P.G. LeFloch, An entropy satisfying MUSCL scheme for systems of conservation laws, Numer. Math. 74 (1996), 1-33. MR 97g:65179

5. P. Colella, A direct Eulerian MUSCL scheme for gas dynamics, SIAM J. Sci. Stat. Comput. 6 (1985), 104-117. MR 86g:65156

6. M.G. Crandall and A. Majda, Monotone difference approximations for scalar conservation laws, Math. of Comp. 34 (1980), 1-21. MR 81b:65079

7. C.M. Dafermos, Generalized characteristics in hyperbolic conservation laws: a study of the structure and the asymptotic behavior of solutions,, in "Nonlinear Analysis and Mechanics: Heriot-Watt symposium", ed. R.J. Knops, Res. Notes in Math., vol. 17, Pitman, London, 1977, pp. 1-58. MR 58:1693

8. B. Engquist and S.J. Osher, One-sided difference approximations for nonlinear conservation laws, Math. Comp. 36 (1981), 321-351. MR 82c:65056

9. A.F. Filippov, Differential equations with discontinuous right-hand side, Mat. Sb. 51 (1960), 99-128; English transl., Amer. Math. Soc. Transl. (2) 42 (1964), 199-231. MR 22:4846

10. J. Glimm and P.D. Lax, Decay of solutions of nonlinear hyperbolic conservation laws, Mem. Amer. Math. Soc 101 (1970). MR 42:676

11. E. Godlewski and P.-A. Raviart, Hyperbolic Systems of Conservation Laws, Math. \& Appl., no. 3/4, Ellipses, Paris, 1991. MR 45i:65146

12. J. Goodman and R. LeVeque, A geometric approach to high resolution TVD schemes, SIAM J. Numer. Anal. 25 (1988), 268-284. MR 89c:65097

13. A. Harten, On a class of high order resolution total-variation-stable finite-difference schemes, SIAM J. Numer. Anal. 21 (1974), 1-23. MR 85f:65085

14. A. Harten, High resolution schemes for hyperbolic conservation laws, J. Comput. Phys. 49 (1983), 357-393. MR 84g:65115

15. A. Harten, B. Engquist, S. Osher and S. Chakravarthy, Uniformly high order accurate essentially non-oscillatory schemes, J. Comput. Phys. 71 (1987), 231-303. MR 90a:65119

16. A. Harten, J.M. Hyman and P.D. Lax, On finite-difference approximations and entropy conditions for shocks, Comm. Pure Appl. Math. 29 (1976), 297-322. MR 54:1640

17. G. Jiang and C.-W. Shu, On cell entropy inequality for discontinuous Galerkin methods, Math. Comp. 62 (1994), 531-538. MR 94h:65099

18. B.K. Quinn (now B.L. Keyfitz), Solutions with shocks, an example of an $L^{1}$ contractive semigroup, Comm Pure Appl. Math. 24 (1971), 125-132. MR 42:6428

19. S.N. Kružkov, First order quasilinear equations in several independent variables, Math. USSR Sbornik 10 (1970), 217-243. MR 42:2159

20. P.D. Lax, Hyperbolic systems of conservation laws II, Comm. Pure Appl. Math. 10 (1957), 537-566. MR 20:176

21. P.D. Lax, Hyperbolic Systems of Conservation Laws and the Mathematical Theory of Shock Waves, SIAM, Philadelphia, 1973. MR 50:2709

22. P.D. Lax and B. Wendroff, Systems of conservation laws, Comm. Pure Appl. Math. 13 (1960), 217-237. MR 22:11523

23. B. van Leer, Towards the ultimate conservative difference scheme, II, Monotonicity and conservation combined in a second order scheme, J. Comp. Phys. 14 (1974), 361-370.

24. B. van Leer, Towards the ultimate conservative difference scheme, V, A second order sequel to Godunov's method, J. Comp. Phys. 32 (1979), 101-136.

25. P.G. LeFloch and J.G. Liu, Discrete entropy and monotonicity criterion for hyperbolic conservation laws, C.R. Acad. Sc. Paris 319 (1994), 881-886. MR 95g:65121

26. A.Y. Leroux, Approximation de quelques problèmes hyperboliques non linéaires, Thèse d'état, Université de Rennes (1979).

27. P.-L. Lions and P. Souganidis, Convergence of MUSCL type methods for scalar conservation laws, C.R. Acad. Sc. Paris, Série I, 311 (1990), 259-264. MR 91i:65168

28. P.-L. Lions and P. Souganidis, Convergence of MUSCL and filtered schemes for scalar conservation laws and Hamilton-Jacobi equations, Numer. Math. 69 (1995), 441-470. MR 96k:65112

29. H. Nessyahu and E. Tadmor, The convergence rate of approximate solutions for nonlinear scalar conservation laws, SIAM J. Numer. Anal. 29 (1992), 1505-1519. MR 93j:65139

30. H. Nessyahu, E. Tadmor, and T. Tamir, The convergence rate of Godunov type schemes, SIAM J. Numer. Anal. 31 (1994), 1-16. MR 94m:65140

31. S.J. Osher, Riemann solvers, the entropy condition and difference approximations, SIAM J. Numer. Anal. 21 (1984), 217-235. MR 86d:65119 
32. S.J. Osher, Convergence of generalized MUSCL schemes, SIAM J. Numer. Anal. 22 (1985), 947-961. MR 87b:65147

33. S.J. Osher and E. Tadmor, On the convergence of difference approximations to scalar conservation laws, Math. of Comp. 50 (1988), 19-51. MR 89m:65086

34. C.W. Shu, Total-variation-diminishing time discretizations, SIAM J. Sci. Stat. Comp. 9 (1988), 1073-1084. MR 90a:65196

35. E. Tadmor, Numerical viscosity and the entropy condition for conservative difference schemes, Math. of Comp. 43 (1984), 369-382. MR 86g:65163

36. E. Tadmor, Convenient total variation diminishing conditions for nonlinear difference schemes, SIAM J. Numer. Anal. 25 (1988), 1002-1014. MR 90b:65173

37. A.I. Volpert, The space $B V$ and quasilinear equations, Mat. Sbornik 73 (1967), 255-302; English transl., Math. USSR Sbornik 2 (1967), 225-267. MR 35:7172

38. H. Yang, On wavewise entropy inequalities for high-resolution schemes I : the semidiscrete case, Math. of Comput. 65 (1996), 45-67. MR 96d:65154

39. H. Yang, On wavewise entropy inequalities for high-resolution schemes II : fully-discrete MUSCL schemes with exact evolution in small time, SIAM J. Numer. Anal., to appear.

Centre de Mathématiques Appliquées and Centre National de la Recherche Scientifique, URA 756, Ecole Polytechnique, 91128 Palaiseau, France

E-mail address: lefloch@cmapx.polytechnique.fr

Department of Mathematics, Temple University, Philadelphia, Pennsylvania 19122

E-mail address: jliu@math.temple.edu 